\title{
Information sharing networks in linear quadratic games
}

\section{Sergio Currarini \& Francesco Feri}

\section{International Journal of Game Theory}

ISSN 0020-7276

Int J Game Theory

DOI 10.1007/s00182-014-0450-x

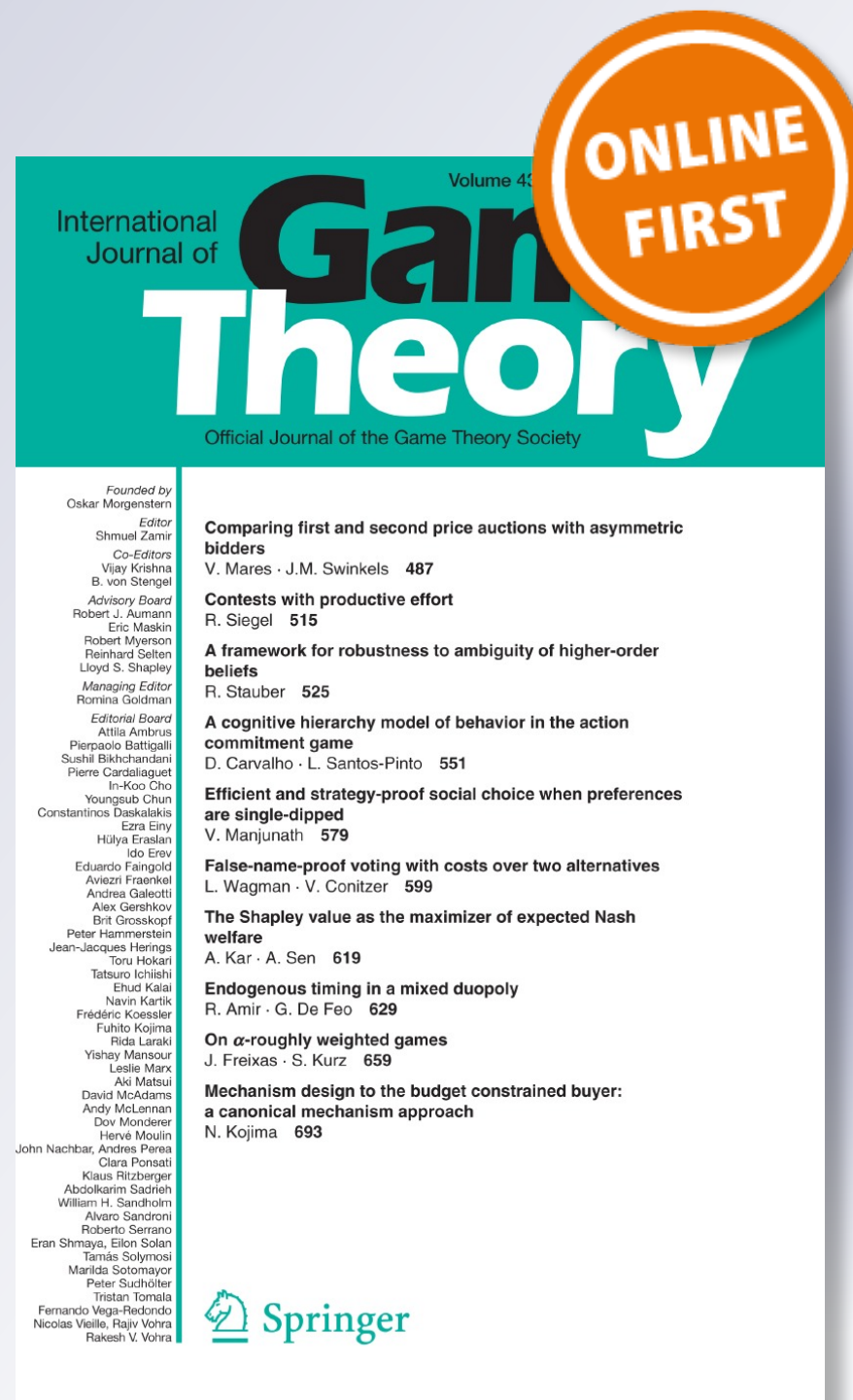

Springer 
Your article is protected by copyright and all rights are held exclusively by SpringerVerlag Berlin Heidelberg. This e-offprint is for personal use only and shall not be selfarchived in electronic repositories. If you wish to self-archive your article, please use the accepted manuscript version for posting on your own website. You may further deposit the accepted manuscript version in any repository, provided it is only made publicly available 12 months after official publication or later and provided acknowledgement is given to the original source of publication and a link is inserted to the published article on Springer's website. The link must be accompanied by the following text: "The final publication is available at link.springer.com". 


\title{
Information sharing networks in linear quadratic games
}

\author{
Sergio Currarini · Francesco Feri
}

Accepted: 10 September 2014

(c) Springer-Verlag Berlin Heidelberg 2014

\begin{abstract}
We study the bilateral exchange of information in the context of linear quadratic games. An information structure is here represented by a non directed network, whose nodes are agents and whose links represent sharing agreements. We first study the equilibrium use of information given the network, finding that the extent to which a piece of information is observed by others affects the equilibrium use of it, in line with previous results in the literature. We then study the incentives to share information ex-ante, highlighting the role of the elasticity of payoffs to the equilibrium volatility of one's own strategy and of opponents' strategies. For the case of uncorrelated signals we fully characterise pairwise stable networks for the general linear quadratic game. For the case of correlated signals, we study pairwise stable networks for three specific linear quadratic games-Cournot Oligopoly, Keynes' Beauty Contest and a Public Good Game-in which strategies are substitute, complement and orthogonal, respectively. We show that signals' correlation favours the transmission of information, but may also prevent all information from being transmitted.
\end{abstract}

Electronic supplementary material The online version of this article (doi:10.1007/s00182-014-0450-x) contains supplementary material, which is available to authorized users.

S. Currarini

University of Leicester, Leicester, UK

e-mail: sc526@le.ac.uk

S. Currarini ( $\square)$

Universita' di Venezia, Venezia, Italy

e-mail: s.currarini@unive.it

F. Feri

Royal Holloway, University of London, Egham, UK

Published online: 02 October 2014 
Keywords Information sharing - Networks - Bayesian equilibrium - Beauty Contest · Oligopoly

JEL Classification D43 $\cdot$ D82 $\cdot$ D85 $\cdot$ L13

\section{Introduction}

Linear quadratic games have played a key role in the analysis of games of incomplete information in economics. The implied linear best reply functions allow for the existence of a Bayesian equilibrium in affine strategies (see Radner 1962; Angelitos and Pavan 2007). Specific examples include linear Cournot Oligopoly (CO), Keynes' Beauty Contest (BC) and Public Good games with linear benefits and quadratic costs (PG). The analytical tractability of linear quadratic games has motivated an extensive theoretical effort to understand the use of information in environments with fundamental uncertainty, tracing such use to strategic features of the game such as complementarity and substitutability, and drawing welfare and policy implications (see Morris and Shin 2002; Angelitos and Pavan 2007).

One issue that is strictly related to the equilibrium use of private information is the possibility that agents share their private information before engaging in non cooperative behaviour. In the framework of imperfect market competition, this issue has spurred an extensive debate that dates back to the seminal contributions of Novshek and Sonnenschein (1982) and Vives (1985). Understanding the incentives of firms to share information before engaging in market competition is important since it can help draw a line between collusive market behaviour (which is suboptimal) and pure sharing of information prior to competition (which is socially desirable). One main insight from this body of literature is that incentives to share are associated with either strategic complementarity or weak substitutability, be it induced by products differentiation, by cost convexity or by price competition (see Vives 1985; Raith 1996). While certainly of great relevance for policy and for welfare, these conclusions rest on the specifics of the imperfect competition model, and little is known about the incentives to share in other instances of linear quadratic games. Moreover, while the traditional approach has mainly studied the incentives of firms to jointly and universally disclose all private information, ${ }^{1}$ in many economic contexts agents may agree to share information in smaller groups or in pairs, by means of private agreements with various degrees of commitment.

These considerations motivate the present analysis of information sharing in general linear quadratic games. We approach information sharing from a bilateral perspective, assuming that each pair of agents can commit (ex-ante) to mutually (and truthfully) disclose their own private information to each other. The ex-ante assumption allows us to dismiss all strategic considerations that relate to the inference of other agents' information from their sharing behaviour. In this context, an information structure is well represented by a non directed network, in which an agent's private information consists of the signals observed by herself and by her "neighbours" in the network.

\footnotetext{
${ }^{1}$ With the exception of Kirby (1988) and Malueg and Tsutsui (1996).
} 
Compared to previous literature where each agent observes a "private" signal (only revealed to her) and a "public" signal (observed by all) (see Morris and Shin 2002; Angelitos and Pavan 2007), here each signal is public to a specific subset of agentsthe neighbourhood.

Agents decide whether to engage in sharing agreements prior to observing their own private signal. Once signals are observed, players play a linear quadratic game of incomplete information, in which information sets are determined by the network. We first characterise the equilibrium use of information in the network. We find that the sensitivity of each player's strategy to each observed signal in the network depends on the strategic nature of the underlying game. In line with previous works in the literature, strategic complementarities induce agents to use more intensively those signals that are observed and used more intensively by other agents in the network. Opposite conclusions apply to games with strategic substitutes.

In Sect. 4 we study the incentives to share information. Our focus is on "pairwise stable" networks, providing no pair of agents with the incentive to form a new link, and no agent with the incentive to unilaterally sever an existing link (see Jackson and Wolinsky (1996)). Differently from all previous works on information sharing in oligopolies, our analysis cannot exploit the symmetry of agents' equilibrium strategies even when the underlying game and the statistical structure are in all respects symmetric. In fact, the gains accruing to an agent severing a link or to two agents forming a new one are assessed by evaluating the (expected) change in payoffs due to a local change in the existing network. Lacking symmetry, the analysis of incentives becomes soon too complex for a comprehensive characterisation of stable networks for all statistical models. Much of the complexity is due to the widespread interrelation of agents' equilibrium use of information, due to the inherent correlation of signals (unconditional and, possibly, conditional).

In Sect. 4.1, as a first tractable benchmark, we study the case of independent signals. This limit case is obtained by setting a conditional correlation which exactly offsets the natural signals' correlation induced by the state of the world. This artificial case, first suggested in Raith (1996), generates a model which is formally equivalent to the model of imperfect competition with i.i.d. signals used in Gal-Or (1985), to which some of our results apply. This approach is quite standard in common value problems such as auctions (see Bulow and Klemperer 2002; Levin 2004; Tan 2012), and has been employed by Hagenbach and Koessler (2010) in their analysis of strategic information transmission in networks (see below for a discussion of the differences between their approach and ours). Using this case as a benchmark will also prove useful in Sect. 4.2 to understand the role of correlation in shaping the incentives to share information. For this case we provide a full characterisation of pairwise stable networks in the general linear quadratic problem. We find that the incentives to share, and the architecture of stable networks, crucially depend on how sensitive payoffs are to the volatility of one's own action, on aggregate volatility, and to the covariance of opponents' actions and the state of the world. When payoffs mostly depend on one's own equilibrium volatility, stable architectures are made of fully connected components of increasing sizes (possibly including singletons); when strategies are complements, only the complete network survives among such structures. When payoffs are also sensitive to aggregate volatility, then incomplete stable structures may emerge, even when strategies are 
complements. In particular, we show that regular incomplete networks can be stable when aggregate volatility is detrimental to one's own payoff. This is somewhat contrary to the common perception that strategic complementarities should provide agents with the incentive to share all of their available information. This perception is correct in the specific case of the BC, where the effect of aggregate volatility is bounded in magnitude, but not in more general frameworks. Finally, we study the case in which payoffs are also sensitive to the covariance of opponents strategies and the state of the world. Here we focus on games in which strategies are orthogonal, and focus on the interplay of the various parameters on the incentives to share and stability. We fully characterise pairwise stable networks, show that such networks can have incomplete architecture, and that stable networks may fail to exist for certain ranges of parameters.

In Sect. 4.2 we finally turn to the case of correlated signals. Here, we focus on three specific cases of the linear quadratic game, each stressing the role of one of the components of the general model. For the $\mathrm{CO}$, where only one's own equilibrium volatility is payoff relevant, we show that two firms may find it profitable to share information when no other firms do. In particular, incentives to share build up when signals are conditionally correlated, and one extra signals provides better inference on the other, unobserved, signals. This "global" improved inference comes without the disclosure of one's own signal to any other firms except for the new sharing partner. When signals' correlation is not too weak, these incentives are sufficient to rule out the pairwise stability of the empty network, for any level of products' differentiation. Moreover, the complete network is pairwise stable for all levels of signals' correlation. We further study how signals' correlation affects the incentives to share information in the context of a four-firm example with no product differentiation, for which we fully characterise pairwise stable networks as a function of signals' correlation. We find that while correlation creates additional incentive to share information, such incentives decrease with the number of observed signals, and may disappear before all information is shared. We then turn to the $\mathrm{BC}$, and show that the complete network is always pairwise stable, while the empty network never is. Finally, for the PG we show that the complete network is the unique pairwise stable information structure.

We finish by commenting on some recent literature on information transmission in networks. Galeotti et al. (2009) study the "many sender-many receivers" game of cheap talk, interpreting the flows of truthful information as directed links in a network. The focus is there on the incentives to truthfully report the observed information, and while in their model all agents would benefit from the disclosure of all available information, this may not be feasible in equilibrium. Hagenbach and Koessler (2010) enrich this basic cheap talk model by adding a coordination motive as in the BC (our Sect. 4.1). They keep the analysis tractable by assuming that the state of the world takes the form of the sum of agents' independent signals, essentially ruling out correlation as we do in Sect. 4.1. Our Proposition 5 (showing that the complete network is the unique pairwise stable structure in the BC) can indeed be viewed as a corollary of their Proposition 2, where it is shown that agents always benefit from disclosing or receiving additional information. Our Proposition 8 (showing that the complete network is a pairwise stable structure), instead, extends the analysis of the BC to correlated signals, while the rest of our analysis, where we study other classes of linear quadratic games, is less related to theirs and closer in spirit to the quoted literature on 
oligopolies. As a general comment, our focus is on the incentives to share information at the ex-ante stage, as they result from the gains from acquiring and the possible losses from disclosing. Since we assume identical preferences, truth-telling is not an issue in the BC, while there is no truth-telling equilibrium in CO (as shown in Ziv 1993).

The paper is organised as follows: Sect. 2 presents the economic and statistical models. Section 3 studies how agents use the available information in the network. Section 4 studies information sharing with and without correlation of signals. Section 5 concludes the paper.

\section{The model}

We consider a set of $n$ agents, each agent $i$ choosing an action $a_{i} \in \Re$. Agent $i^{\prime} s$ utility is given as a function of her action $a_{i}$, the sum of other agents' actions $A_{i}=\sum_{j \neq i} a_{j}$ and a parameter $\theta$ denoted as the "state of the world":

$$
u_{i}\left(a_{i}, A_{i}, \theta\right)=\left(\begin{array}{c}
\lambda_{a} \\
\lambda_{A} \\
\lambda_{\theta}
\end{array}\right)^{\prime}\left(\begin{array}{c}
a_{i} \\
A_{i} \\
\theta
\end{array}\right)+\left(\begin{array}{c}
a_{i} \\
A_{i} \\
\theta
\end{array}\right)^{\prime}\left(\begin{array}{ccc}
\gamma_{a} & \gamma_{a A} & \gamma_{a \theta} \\
\gamma_{a A} & \gamma_{A} & \gamma_{A \theta} \\
\gamma_{a \theta} & \gamma_{A \theta} & \gamma_{\theta}
\end{array}\right)\left(\begin{array}{c}
a_{i} \\
A_{i} \\
\theta
\end{array}\right)
$$

where the $\gamma$ coefficients in the interaction matrix measure the quadratic relations between agents' utilities, agents' actions and the state of the world, while the $\lambda$ coefficient measure the linear relations between agents' utilities, agents' actions and the state of the world.

The state of the world $\theta$ is assumed to be a random variable of the form

$$
\theta=\mu_{\theta}+\epsilon
$$

where $\epsilon \sim N(0, t)$ and $\mu_{\theta}$ is a constant and its value is common knowledge. Agents' information is structured as follows. Each agent $i$ receives a private noisy signal $y_{i}$ about $\epsilon$, with

$$
y_{i}=\epsilon+\eta_{i}
$$

where $\eta_{i} \sim N(0, u)$ for all $i$, and where $\operatorname{cov}\left(\eta_{i}, \eta_{j}\right)=u_{n}$ for all $i, j$. We also assume that $\operatorname{cov}\left(\eta_{i}, \epsilon\right)=0$ for all $i$. For notational convenience, we will denote by $p_{s}=(t+u)$ the variance of signals and by $p_{n}=\left(t+u_{n}\right)$ the covariance of signals.

The quasilinear model has many application in different fields of economic analysis. Here there are three such examples.

Beauty Contest (BC) (Morris and Shin 2002; Hagenbach and Koessler 2010). There are $n$ agents, each setting an action $a_{i}$. Each agent $i$ suffers a loss which increases quadratically with the distance between her action and the average action chosen by the opponents, and a loss which increases quadratically with the distance between her action and the realisation of a random state of the world $\theta$ :

$$
u_{i}\left(a_{i}, A_{i}, \theta\right)=-v\left(a_{i}-\theta\right)^{2}-(1-v)\left(a_{i}-\frac{A_{i}}{n-1}\right)^{2} .
$$


Using the notation of the present paper, we have: $\gamma_{a}=-1 ; \gamma_{\theta}=-v ; \gamma_{a \theta}=2 v$; $\gamma_{A}=-\frac{(1-v)}{(n-1)^{2}} ; \gamma_{a A}=\frac{2(1-v)}{n-1}$. All other coefficients are zero.

Cournot Oligopoly (CO) (Vives 1985; Kirby 1988; Raith 1996). There are $n$ firms competing in a common market with inverse demand function:

$$
p=\theta-a_{i}-\delta A_{i}
$$

where $a_{i}$ denotes firm $i$ 's output, $A_{i}$ denotes the aggregate output of $i$ 's competitor, and $\delta$ captures product differentiation. Firms produce with no costs. We have: $\gamma_{a}=-1 ; \gamma_{a \theta}=1 ; \gamma_{a A}=-\delta$. All other coefficients are zero.

Public goods (PG) (Ray and Vohra 1999). Each agent $i$ contributes the amount $a_{i}$ to a global public good, and has utility function:

$$
u_{i}\left(A_{i}, a_{i}\right)=\theta\left(A_{i}+a_{i}\right)-b a_{i}^{2} .
$$

The actual value of the public good is a random variable $\theta$ about which each agent receives a noisy signal. We have: $\gamma_{a}=-b ; \gamma_{A \theta}=1 ; \gamma_{a \theta}=1$. All other coefficients are zero.

We will consider the possibility that agents share their information by means of bilateral and truthful sharing agreements; this means that agent $i$ is allowed to observe agent $j$ 's signal if and only if he reveals his own signal to agent $j$. Sharing agreements need not be transitive, in the sense that information sharing between agents $i$ and $j$ and between agents $j$ and $k$ need not imply information sharing between agents $i$ and $k$. In this sense, the information structure induced by such agreements may differ from a collection of information sharing coalitions, and is well represented by a non directed network $g$, in which the set of nodes is the set of agents, and a link $i j$ denotes a bilateral agreement between agents $i$ and $j$. We denote by $N_{i}(g) \equiv\{j: i j \in g\} \cup\{i\}$ the set of neighbours of $i$ in $g$ (including $i$ ) and we denote by $n_{i}^{g}=\left|N_{i}(g)\right|$ the number of such neighbours. In other words, $n_{i}^{g}$ is the number of signals observed by $i$ in $g$ (in the terminology of graph theory, this number is the degree of agent $i$ in $g$ augmented by one). The information available to agent $i$ in the network $g$ is therefore $I_{i}(g) \equiv\left\{y_{j}: j \in N_{i}(g)\right\}$. We will use the notation $g+i j$ to denote the network obtained by adding to $g$ the link $i j \notin g$, and $g-i j$ to denote the network obtained by severing the link $i j \in g$ from $g$. A component $h$ of the network $g$ is a network with set of nodes $N(h) \subset N$ and such that: i) $i j \in h$ if and only if $i j \in g$ and; ii) if $i \in N(h)$ and $i k \in g$ then $k \in N(h)$. The network $g$ is fully connected if $i j \in g$ for all $i \in N$ and $j \in N$.

\section{Use of information in the network}

With each possible information structure $g$ we associate the Bayesian Nash equilibrium of the game in which each agent $i$ sets her action $a_{i}$ in order to maximise her expected payoff, given the available information-determined by $i$ 's links in $g$-and given the optimal decisions of the other agents. Formally, a Bayesian Nash equilibrium associ- 
ated with $g$ is a family of functions $a_{i}^{g}$ mapping, for each $i \in N$, the available information $I_{i}(g)$ into a choice $a_{i}^{g}\left(I_{i}(g)\right)$ satisfying for each agent $i$ the following condition:

$$
a_{i}^{g}\left(I_{i}(g)\right)=\arg \max _{a_{i} \in \Re} E\left[u_{i}\left(a_{i}, A_{i}^{g}\left(I_{-i}(g)\right), \theta \mid I_{i}(g)\right]\right.
$$

where $A_{i}^{g}\left(I_{-i}(g)\right)$ is the sum of strategies of all agents but $i$, and

$$
I_{-i}(g) \equiv\left(I_{1}(g), \ldots, I_{i-1}(g), I_{i+1}(g), \ldots, I_{n}(g)\right) .
$$

Note that the terms $\gamma_{A}, \gamma_{A \theta}, \lambda_{A}, \lambda_{\theta}, \gamma_{\theta}$ do not affect the equilibrium strategies; they however affect welfare, and will be therefore relevant in determining agents' incentives to form links. The necessary first order condition for (2) is:

$$
a_{i}^{g}\left(I_{i}(g)\right)=-\frac{\lambda_{a}+\gamma_{a \theta} E\left[\theta \mid I_{i}(g)\right]+\gamma_{a A} E\left[A_{i}^{g}\left(I_{-i}(g)\right) \mid I_{i}(g)\right]}{2 \gamma_{a}}
$$

As in Angelitos and Pavan (2007), we will assume $\gamma_{a}<0$ and $\gamma_{a}+(n-1) \gamma_{a A}<0$. Standard results (see Radner 1962; Angelitos and Pavan 2007) can be used to establish the existence of a unique Bayesian Nash Equilibrium for all information structures $g$, with the equilibrium strategies affine in the observed signals, i.e.:

$$
a_{i}^{g}\left(I_{i}(g)\right)=\alpha_{i}^{g}+\sum_{j \in N_{i}(g)} \beta_{i j}^{g} y_{j}, \quad i=1,2, \ldots n .
$$

The following proposition derives the system defining the equilibrium $\alpha_{i}^{g}$ and $\beta_{i j}^{g}$ coefficients in the Bayesian game with information structure $g$.

Proposition 1 The Bayesian Nash equilibrium of the game with payoff functions (1) and information structure described by the network $g$ is characterised by the following system:

$$
\begin{aligned}
\alpha_{i}^{g}= & -\frac{1}{2 \gamma_{a}}\left(\lambda_{a}+\gamma_{a \theta} \mu_{\theta}+\gamma_{a A} \sum_{j \neq i} \alpha_{j}^{g}\right), \quad i=1,2, \ldots n \\
\beta_{i h}^{g}= & -\frac{1}{2 \gamma_{a}}\left(\gamma_{a \theta} k_{1}^{i g}+\gamma_{a A}\left(\sum_{j \in N_{h}(g) \backslash i} \beta_{j h}^{g}+\sum_{z \notin N_{i}(g)} \sum_{j \in N_{z}(g)} k_{2}^{i g} \beta_{j z}^{g}\right)\right), \\
& \forall h \in N_{i}, \quad i=1,2, \ldots n
\end{aligned}
$$

where

$$
k_{1}^{i g}=\frac{t}{p_{s}+\left(n_{i}^{g}-1\right) p_{n}} ; \quad k_{2}^{i g}=\frac{p_{n}}{p_{s}+\left(n_{i}^{g}-1\right) p_{n}}
$$


are the updating coefficients that agent $i$ applies to each $y_{j} \in I_{i}(g)$ to take the following expectation on the state $\theta$ and of the signals $y_{h}, h \notin N_{i}(g)$, respectively:

$$
\begin{aligned}
E\left[\theta \mid I_{i}(g)\right] & =\mu_{\theta}+k_{1}^{i g} \sum_{j \in N_{i}(g)} y_{j} ; \\
E\left[y_{h} \mid I_{i}(g)\right] & =k_{2}^{i g} \sum_{j \in N_{i}(g)} y_{j} .
\end{aligned}
$$

From Proposition 1 we obtain a first insight in how the information structure $g$ affects the way in which agents use their available information. First, from (5) it is directly verifiable that the coefficient $\alpha_{i}^{g}$ is the same in all networks and for all agents, which allows us to denote $\alpha_{i}^{g}=\alpha$.

Expression (6) describes the way in which information is used in equilibrium as a function of the whole network. To fix ideas, assume that $\gamma_{a \theta}>0$, so that agents' choices move together with the state of the world. The coefficient that $i$ applies to signal $y_{h} \in I_{i}(g)$ is equal to the sum of the term $\left(-\frac{\gamma_{a} \theta}{2 \gamma_{a}} k_{1}^{i g}\right)$ and of the two summations in the second bracket of (6) multiplied by $\left(-\frac{\gamma_{a A}}{2 \gamma_{a}}\right)$. Both summations amplify the effect of the first term if actions are strategic complements $\left(\gamma_{a A}>0\right)$, and weaken the effect of the first term if strategies are instead substitutes $\left(\gamma_{a A}<0\right)$. Both summations measure the reactions of $i$ 's opponents that are correlated to signal $y_{h} \in I_{i}(g)$; the first refers to the reaction of the opponents that observe $y_{h} \in I_{i}(g)$, the second to those signals that agent $i$ does not observe, but that are correlated to signal $y_{h} \in I_{i}(g)$. Both terms tend to amplify the use of signal $y_{h} \in I_{i}(g)$ by agent $i$ when there is an incentive to correlate with other agents (complements), and to reduce the use of signal $y_{h} \in I_{i}(g)$ when the incentive is to diversify from the other agents (substitutes). We identify a congestion effect in the use of information: agents tend to use less (more) those pieces of information that are more intensively used by other agents when strategies are substitutes (complements). This is formally stated in the following proposition, directly implied by (6).

Proposition 2 For all $i h \in g$ and $i j \in g$ :

$$
\beta_{i h}^{g}-\beta_{i j}^{g}=\frac{\gamma_{a A}}{2 \gamma_{a}}\left(\sum_{k \in N_{j}(g) \backslash i} \beta_{k j}^{g}-\sum_{k \in N_{h}(g) \backslash i} \beta_{k h}^{g}\right) .
$$

The next example illustrates the equilibrium use of information in a network where agents' positions present stark differences - the star.

Example 1 Consider the star network $g^{s}$ with 4 agents, where the central agent $i$ receives a signal $y_{i}$ which is observed by all agents, and each other signal $y_{h}$ is observed by the receiving agent $h$ and by the central agent $i$. We obtain the following coefficients for the cases of strategic complements $\left(\gamma_{a A}=.1\right)$ and substitutes $\left(\gamma_{a A}=-1\right)$, and for different levels of signals' correlation (see Appendix 2 for detailed expressions of the coefficients). 


\begin{tabular}{lllll}
\hline & $p_{n}=.6 ; \gamma_{a A}=-1$ & $p_{n}=.8 ; \gamma_{a A}=-1$ & $p_{n}=.6 ; \gamma_{a A}=.1$ & $p_{n}=.8 ; \gamma_{a A}=.1$ \\
\hline$\beta_{i j}^{g}$ & 0.014 & 0.009 & 0.117 & 0.099 \\
$\beta_{i h}^{g}$ & 0.047 & 0.037 & 0.097 & 0.081 \\
$\beta_{h h}^{g}$ & 0.083 & 0.071 & 0.171 & 0.153 \\
$\beta_{h i}^{g}$ & 0.050 & 0.042 & 0.191 & 0.171 \\
\hline
\end{tabular}

We see that signal $y_{i}$, which is observed by all agents and is therefore a public signal, is used less intensively under strategic substitutes, and more intensively under complements, than signals $y_{h}$, observed only by two agents. Moreover, periphery agents, who are endowed with fewer pieces of information, use their information more intensively. Signals' correlation has a negative impact on the use of information under both substitutes and complements.

\section{Information sharing}

We study the incentives to share information at the ex-ante stage. For each network $g$, we denote by $u_{i}^{e}(g)$ the ex-ante expected utility for agent $i$, given that $g$ describes the information structure of the Bayesian game played at the interim stage. The utility $u_{i}^{e}(g)$ is obtained by taking the expectation of the interim utility $E\left[u_{i} \mid I_{i}(g)\right]$ over all possible realisations of $i$ 's information $I_{i}(g)$. The interim utility is given by:

$$
\begin{aligned}
E\left[u_{i} \mid I_{i}(g)\right]= & \lambda_{a} a_{i}^{g}\left(I_{i}(g)\right)+\lambda_{A} E\left[A_{i}^{g} \mid I_{i}(g)\right]+\lambda_{\theta} E\left[\theta \mid I_{i}(g)\right]+\gamma_{a} a_{i}^{g}\left(I_{i}(g)\right)^{2}+ \\
& +\gamma_{A} E\left[\left(A_{i}^{g}\right)^{2} \mid I_{i}(g)\right]+\gamma_{\theta} E\left[\theta^{2} \mid I_{i}(g)\right]+\gamma_{a A} a_{i}^{g} E\left[A_{i}^{g} \mid I_{i}(g)\right]+ \\
& +\gamma_{a} a_{i}^{g}\left(I_{i}(g)\right) E\left[\theta \mid I_{i}(g)\right]+\gamma_{A \theta} E\left[A_{i}^{g} \theta \mid I_{i}(g)\right] .
\end{aligned}
$$

Together with the first order condition (3), (10) yields the following expression:

$$
\begin{aligned}
& E\left[u_{i} \mid I_{i}(g)\right]=-\gamma_{a} \cdot a_{i}^{g}\left(I_{i}(g)\right)^{2}+\lambda_{A} \cdot E\left[A_{i}^{g} \mid I_{i}(g)\right]+\lambda_{\theta} E\left[\theta \mid I_{i}(g)\right]+ \\
& +\gamma_{A} \cdot E\left[\left(A_{i}^{g}\right)^{2} \mid I_{i}(g)\right]+\gamma_{\theta} \cdot E\left[\theta^{2} \mid I_{i}(g)\right]+\gamma_{A \theta} \cdot E\left[A_{i}^{g} \theta \mid I_{i}(g)\right] .
\end{aligned}
$$

Given the linear specification of equilibrium strategies in (4), we can express the ex-ante equilibrium utility in any given network $g$ as follows:

$$
\begin{aligned}
u_{i}^{e}(g)= & \lambda_{A} \cdot(n-1) \cdot \alpha+\alpha^{2} \cdot\left(\gamma_{A} \cdot(n-1)^{2}-\gamma_{a}\right)+\left(\lambda_{\theta}+\gamma_{\theta} \mu_{\theta}\right) \mu_{\theta}+\gamma_{A \theta} . \\
& (n-1) \cdot \alpha \cdot \mu_{\theta}+\gamma_{\theta} \cdot \operatorname{var}(\theta)+\gamma_{A} \cdot \operatorname{var}\left(A_{i}^{g}\right)+\gamma_{A \theta} \cdot \operatorname{cov}\left(A_{i}^{g}, \theta\right) \\
& -\gamma_{a} \cdot \operatorname{var}\left(a_{i}^{g}\right) .
\end{aligned}
$$

where the variances and covariance of equilibrium strategies are:

$$
\operatorname{var}\left(a_{i}^{g}\right)=\sum_{h \in N_{i}^{g}}\left(\beta_{i h}^{g}\right)^{2} p_{s}+2 \sum_{h \in N_{i}} \sum_{k<h} \beta_{i h}^{g} \beta_{i k}^{g} p_{n}
$$




$$
\begin{aligned}
\operatorname{var}\left(A_{i}^{g}\right)=p_{s} \cdot & {\left[\sum_{j \in N}\left(B_{i j}^{g}\right)^{2}\right]+p_{n} \cdot\left[2 \cdot \sum_{j \in N} \sum_{k<j} B_{i j}^{g} \cdot B_{i k}^{g}\right] ; } \\
& \operatorname{cov}\left(A_{i}^{g}, \theta\right)=t \cdot \sum_{j \in N} B_{i j}^{g},
\end{aligned}
$$

where we have denoted by $B_{i j}^{g} \equiv \sum_{h \in N_{j} \backslash i} \beta_{h j}^{g}$ the aggregate reaction to signal $j$ by $i$ 's opponents. Looking at the difference $\left[u_{i}^{e}\left(g^{\prime}\right)-u_{i}^{e}(g)\right]$, we can express the condition under which agent $i$ has an incentive to induce network $g^{\prime}$ from a network $g$ as follows:

$$
\begin{aligned}
& {\left[u_{i}^{e}\left(g^{\prime}\right)-u_{i}^{e}(g)\right]=\gamma_{A} \cdot\left[\operatorname{var}\left(A_{i}^{g^{\prime}}\right)-\operatorname{var}\left(A_{i}^{g}\right)\right]+\gamma_{A \theta} \cdot\left[\operatorname{cov}\left(A_{i}^{g^{\prime}}, \theta\right)\right.} \\
& \left.-\operatorname{cov}\left(A_{i}^{g}, \theta\right)\right]-\gamma_{a} \cdot\left[\operatorname{var}\left(a_{i}^{g^{\prime}}\right)-\operatorname{var}\left(a_{i}^{g}\right)\right]>0
\end{aligned}
$$

The difference in (16) is the sum of three terms, expressing the change, when passing from $g$ to $g^{\prime}$, in the variance of other agents actions, of $i$ 's action and in the covariance of the other agents' actions and the state of the world. Inspection of condition (16) provides insights into the incentives of a generic agent $i$ to induce a given network $g^{\prime}$ from a network $g$. The first term measures the effect of the change in the variance of the aggregate actions of other agents, keeping all other things equal. This effect is weighted by the coefficient $\gamma_{A}$, measuring the effect on utility of the square of other agents' actions (see (1)). This coefficient is null in CO and in PG, and is negative in the $\mathrm{BC}$ with complements. The second term in (16) measures the effect of a change in the covariance of the other agents' actions and the state of the world; this effect is weighted by the coefficient $\gamma_{A \theta}$, controlling in (1) for the interaction of $i$ 's opponents' actions and the state of the world. This is non null in PG. The last term in (16) measures the incentives coming from a change in agent $i$ 's variance in equilibrium, and is weighted by the own quadratic coefficient $\gamma_{a}<0$, implying that increased variance of one's own action is desirable in all linear quadratic games.

We will study the structure of pairwise stable networks (see Jackson and Wolinsky 1996), in which no pair of agents has an incentive to form a new link and no agent has an incentive to unilaterally sever an existing link:

Definition 1 The network $g$ is pairwise stable at the ex-ante stage if: a) $u_{i}^{e}(g+i j)>$ $u_{i}^{e}(g) \Rightarrow u_{j}^{e}(g+i j)<u_{j}^{e}(g)$ for all $i j \notin g$; b) $u_{i}^{e}(g) \geq u_{i}^{e}(g-i j)$ for all $i j \in g$.

A pairwise stable network is here interpreted as an information structure that results from long-run information sharing arrangements, with the property that no additional arrangement occurs and no existing arrangement is discontinued.

\subsection{Information sharing with uncorrelated signals}

We start by studying the case of uncorrelated signals, i.e. of $p_{n}=0$. Formally, this requires that signals' errors are negatively correlated, and that this correlation exactly outweighs the correlation induced by the state of the world: $u_{n}=-t$. Although this 
is a special case of signals' correlation, it is of interest here for two reasons. First, it allows us to better understand in the next section the role of signals' correlation, and of the associated strategic inference, for link formation. Second, uncorrelated signals are of interest in a model where the state of the world is the sum (or the average) of agents' signals, as in Gal-Or (1985), in Hagenbach and Koessler (2010) and in most papers dealing with common value problems in auctions, as, for instance, Levin (2004).

Uncorrelated signals imply that $k_{1}^{i g}=\frac{t}{p_{s}}$ and $k_{2}^{i g}=0$. Equilibrium coefficients simplify as follows:

$$
\begin{aligned}
\alpha & =-\frac{\lambda_{a}+\gamma_{a \theta} \mu_{\theta}}{2 \gamma_{a}+\gamma_{a A}(n-1)} ; \\
\beta_{i h}^{g} & =-\frac{\gamma_{a \theta} \frac{t}{p_{s}}}{2 \gamma_{a}+\gamma_{a A}\left(n_{h}^{g}-1\right)}, \quad \forall h \in N_{i}^{g} .
\end{aligned}
$$

Note that the coefficient $\beta_{i h}^{g}$ applied by agent $i$ to the piece of information $y_{h}$ only depends on how many other agents observe $y_{h}$, and on no other feature of the network. This substantially simplifies the analysis of agents' incentives to form and sever a link starting from an arbitrary network $g$. In particular, we will see in the results to follow that the incentives of an agent $i$ to form the link $i j \notin g$, as expressed by condition (16), only depend on the number of agents observing $y_{i}$ and on the number of agents observing $y_{j}$ in $g$.

Our analysis refers to different classes of games, each corresponding to different assumptions on the three key parameters $\gamma_{A}, \gamma_{a A}$ and $\gamma_{A \theta}$. These classes include the three games $\mathrm{CO}, \mathrm{BC}$ and $\mathrm{PG}$ outlined in our examples.

We start with the case $\gamma_{A}=\gamma_{A \theta}=0$, covering $\mathrm{CO}$ as a special case. From now on we will denote by $\mu \equiv \frac{\gamma_{a A}}{\gamma_{a}}$ the relative strategic interdependence in the game. Note that $\mu<0$ under strategic complements and $\mu>0$ under substitutes. The next preparatory lemma characterises the way in which the incentives to form or maintain a link depend on the degrees of the two involved nodes. We show that when $\mu>0$, this relation involves a threshold degree, below which an agent's information is "attractive" and below which it is not. This is due to a congestion effect similar to the one behind Proposition 2: pieces of information that are observed by many agents are little attractive under strategic substitutes. Moreover, as point (i) below shows, this congestion affects more intensively the incentives of less connected nodes (see Appendix 1 for the proof of this lemma and of all other results).

Lemma 1 Let $p_{n}=\gamma_{A}=\gamma_{A \theta}=0$ and let $\mu>0$. There exist functions $f_{\mu}: \mathbb{N} \rightarrow \mathbb{R}$ and $F_{\mu}: \mathbb{N} \rightarrow \mathbb{R}$ such that: 1) $\left.u_{i}(g+i j)>u_{i}(g) \Longleftrightarrow n_{j}^{g}<f_{\mu}\left(n_{i}^{g}\right): 2\right)$ $u_{i}(g)>u_{i}(g-i j) \Longleftrightarrow n_{j}^{g}<F_{\mu}\left(n_{i}^{g}\right)$. Moreover, these functions satisfy the following properties:

(i) $f_{\mu}$ and $F_{\mu}$ are both increasing;

(ii) $f_{\mu}(m)>m$ for all $2 \leq m ; f_{\mu}(1)>1$ iff $\mu<2(\sqrt{2}-1)$.

(iii) $f_{\mu}(m-1)=F_{\mu}(m)-1$.

(iv) $f_{\mu}(m)>F_{\mu}(m)$. 
The next proposition provides a full characterisation of pairwise stable networks for the case $\gamma_{A}=\gamma_{A \theta}=0$. In a nutshell: under strategic complements the complete network is the unique stable information structure; under substitutes, information is shared in disjoint "groups" (fully connected components), within which information disclosure is complete. These groups are of increasing sizes, and the size differences are governed by the $f_{\mu}$ function defined in Lemma 1. Intuitively, if the sizes of two disjoint components were too close, then agents would have an incentive to bridge these components by means of a new link, and stability would fail.

Proposition 3 Let $p_{n}=0$ and $\gamma_{A}=\gamma_{A \theta}=0$.

1. If $\mu<0$ the unique pairwise stable network is the complete network.

2. If $\mu>0$ then the network $g$ is pairwise stable if and only if it has the following architecture: a set $S$ of isolated nodes and the complement set of nodes $N \backslash S$ organised in fully connected components $\left\{h_{1}, h_{2}, \ldots, h_{m}\right\}$ such that $n\left(h_{i}\right) \geq f_{\mu}\left(n\left(h_{i-1}\right)\right)$ for all $1<k \leq m$. If $0<\mu<\frac{2}{1+\sqrt{2}}$ all pairwise stable networks contain at most one isolated node.

The next corollary draws implications of Proposition 3 for the $\mathrm{CO}$ with i.i.d. signals studied in Gal-Or (1985).

Corollary 1 Consider a Cournot Oligopoly with i.i.d. signals about the demand intercept (Gal-Or 1985), and assume that firms share information by means of bilateral quid-pro-quo agreements. In a pairwise stable information structure, information sharing is organised in coalitions of firms. Within each coalition all information is disclosed to all members; moreover, coalitions have strictly increasing sizes.

Note that while Gal-Or (1985)'s conclusion that firms do not exchange private information refers to the lack of incentives of all firms to coordinate and universally disclose information, our result refers to the incentives of firms to form and sever bilateral agreements. While firms still prefer the complete absence of information sharing (empty network) to the universal disclosure of all information (complete network), pairs of firms would not find it profitable to discontinue an ongoing agreement, or to form a new one, within the stable configuration outlined in Proposition 3.

In the next proposition we turn to the case in which payoffs depend directly on the variance of opponents' actions in equilibrium $\left(\gamma_{A} \neq 0\right)$. We show that when such variance is beneficial, then the complete network is the unique pairwise stable network under strategic complementarity.

Proposition 4 Let $\gamma_{A}>0, \gamma_{A \theta}=0$ and $0<\gamma_{a A}<-\frac{\gamma_{a}}{n-1}$. Then the complete network is the unique pairwise stable network.

This result is explained by the fact that when complementarities are small, then an additional link formed by player $i$ always increases the volatility of $i$ 's own equilibrium strategy and of the sum of all other players' equilibrium strategies. When this increased volatility has a positive effect on $i$ 's payoff $\left(\gamma_{A}>0\right)$, then this positive effect sums up with the positive effect of $i$ 's own increased variability.

Let us now turn to the case in which opponents' volatility is detrimental to an agent's payoff $\left(\gamma_{A}<0\right)$. Here, an additional link $i j$ that results in increased variance in the 
opponents' strategies needs not increase $i$ 's payoff, even if strategies are complements. One game in which strategies are complements and $\gamma_{A}<0$ is the BC, where if $v<1$ we have $\gamma_{a A}=\frac{2(1-v)}{n-1}>0$ and $\gamma_{A}=-\frac{1-v}{(n-1)^{2}}<0$. The next proposition shows that in this game, the negative effect of opponents' increased volatility never outweighs the effect of one's own volatility, and all links form in equilibrium.

Proposition 5 In Keynes' Beauty Contest with strategic complements $(0<v<1)$ the complete network is the unique pairwise stable information structure.

The restrictions imposed by the BC on the parameters $\left(2 \gamma_{A}=-\frac{\gamma_{a A}}{n-1}\right)$ ensure that the incentives to form a new link always remain positive. Once such restrictions are dropped (and, in particular, once $\gamma_{A}$ can grow in magnitude fixing the other parameters), incomplete networks may arise even in the presence of strategic complementarities. The next example illustrates this possibility in a variation of the $\mathrm{BC}$, in which agents try to match the state of the world and to exceed the average of their opponents' strategies by a factor $k$. For this game we show that incomplete networks arise in equilibrium.

Example 2 Consider an economy with ten agents, each having the following payoff function:

$$
u_{i}\left(a_{i}, A_{i}, \theta\right)=-v\left(a_{i}-\theta\right)^{2}-(1-v)\left(a_{i}-k \frac{A_{i}}{n-1}\right)^{2} .
$$

Set $k=1, \gamma_{a A}=\frac{2}{19}$ and $\gamma_{a}=-1$, so that our condition $\gamma_{a}+(n-1) \gamma_{a A}<0$ holds. These assumptions imply $v=0.53$ and $\gamma_{A}=-0.0058$. Computations based on condition (16) show that any two nodes with degree $x<10$ always have an incentive to form a link. This is simply a consequence of Proposition 5 where the complete network is shown to be the unique pairwise stable network in the BC. Consider now values of $k>1$, setting all other parameters as above, we obtain $v=\frac{19 k-9}{19 k}$ and then $\gamma_{A}=-0.0058 \cdot k$. Computations show that for $k$ large enough, incomplete networks can be pairwise stable. For example, when $k=5$ we obtain that (1) a regular network with average degree of 8 is pairwise stable; (2) networks with one fully connected component of nine nodes and one isolated node or two fully connected components of two and eight nodes are pairwise stable.

We finally turn the case in which both parameters $\gamma_{A}$ and $\gamma_{A \theta}$ need not be null. Here we will only focus on games with orthogonal strategies $\left(\gamma_{a A}=0\right)$, and concentrate on the interplay of the parameters $\gamma_{A}, \gamma_{a \theta}$ and $\gamma_{A \theta}$. As a specific case we have PG, where $\gamma_{A \theta}$ is equal to 1 and $\gamma_{A}=0$.

Proposition 6 Let $\gamma_{a A}=0$ and $\gamma_{a \theta}>0$.

1. When $\gamma_{A}=0$, the unique pairwise stable network is either the complete network (iff $2 \gamma_{A \theta}+\gamma_{a \theta}>0$ ) or the empty network (iff $2 \gamma_{A \theta}+\gamma_{a \theta}<0$ ).

2. When $\gamma_{A}>0$ : 
(a) if $2 \gamma_{A \theta}+\gamma_{a \theta}>0$ then the complete network is the unique pairwise stable architecture;

(b) if $2 \gamma_{A \theta}+\gamma_{a \theta}<0$ then there exists $m \leq n$ such that the set of pairwise stable networks consists of all networks made of a fully connected component of $q$ nodes and of $n-q$ isolated nodes, with $q \geq m$, together with the empty network.

3. When $\gamma_{A}<0$ :

(a) if $2 \gamma_{A \theta}+\gamma_{a \theta}>0$, either $1<\frac{2 \gamma_{A \theta}+\gamma_{a \theta}}{\gamma_{a \theta}}$, in which case the empty network is the unique pairwise stable architecture, or otherwise no pairwise stable network exists;

(b) if $2 \gamma_{A \theta}+\gamma_{a \theta}<0$, only the empty network is pairwise stable.

To get some intuition for Proposition 6, note that in the class of games it covers the effect of a change in the variability of opponents' strategies resulting from one additional link is positive when $\gamma_{A}>0$ and negative when $\gamma_{A}<0$, and it increases in magnitude with the degree of the agent forming the link. The term $2 \gamma_{A \theta}+\gamma_{a \theta}$ measures the net gains from the new link as a result of the change in the covariance of opponents' strategies and the state of the world and in one's own strategy. If both $\gamma_{A}$ and $2 \gamma_{A \theta}+\gamma_{a \theta}$ are positive, then all links form. If they have opposite signs, then the prevailing effect will depend on the relative strengths of these weights and on the degree of the agent forming the link. In particular, when covariance is detrimental to utility, then links form only if the (positive) effect of the increased opponents' volatility is large enough, that is for large enough $\gamma_{A}>0$ and for large enough degrees.

As an illustration of this second case, the next example studies a game in which agents wish to guess the state of the world, with a reward that increases the worse is the opponents' guess.

Example 3 Each agent $i$ has the following utility from her guess $a_{i}$ and the opponents' guess $A_{i}$ :

$$
u_{i}=-\left(a_{i}-\theta\right)^{2}+v\left(\frac{A_{i}}{n-1}-\theta\right)^{2}
$$

We have $\gamma_{a}=-1, \gamma_{A \theta}=-2 \frac{v}{(n-1)}, \gamma_{a \theta}=2$, and $\gamma_{A}=\frac{v}{(n-1)^{2}}$. From condition (16) and the fact that $\gamma_{a A}=0$, agent $i$ forms a link $i j$ if and only if the following condition holds:

$$
-\frac{v}{(n-1)^{2}}\left(2 n_{i}^{g}-1\right)<1-2 \frac{v}{(n-1)}
$$

or

$$
\left(2 n_{i}^{g}-1\right)>2(n-1)-\frac{(n-1)^{2}}{v}
$$

For $v=2$ and $n=3$ we have that agent $i$ wishes to form the link $i j$ if and only if $n_{i}^{g} \geq 2$. This implies that there are three pairwise stable architectures in this example: the empty network, the complete network, and the network made of component of 
two connected nodes and one isolated node. For $v=4$ the complete network remains the unique stable network, together with the empty network. If $v$ increases further, the empty network remains the unique stable architecture.

The following corollary applies to the PG, where $\gamma_{A}=0$ and $2 \gamma_{A \theta}+\gamma_{a \theta}=3$.

Corollary 2 In the public good game with linear benefits and quadratic costs, the unique pairwise stable network is the complete network.

\subsection{Information sharing with correlated signals}

We now turn to the case of correlated signals $\left(p_{n}>0\right)$. Here equilibrium computations become complex, due to the potential asymmetry of network structures and of the associated Bayesian Nash equilibria. For this reason we will not provide a full characterisation of pairwise stable networks, but rather investigate the effect of signals' correlation, and in particular of conditional correlation, on the incentives to share information. In particular we show in Proposition 7 that enough correlation guarantees that some positive amount of information is always shared in equilibrium-in the present terminology, that the empty network fails to be pairwise stable. As we shall see, this is due to the strategic advantage that the bilateral information sharing provides in the form of a better inference of other firms' actions. Incentives to form link, however, can die out before all information is shared, as we illustrate by means of a four firm example.

We start with the case of strategic substitutes $(\mu>0)$, assuming that only the volatility of one's own strategy is payoff relevant. This includes $\mathrm{CO}$ as a special case.

Proposition 7 Let $\gamma_{A}=\gamma_{A \theta}=0$ and $\mu>0$. Then:

\section{Under the following conditions the empty network is not pairwise stable:}

(i) $\mu<\frac{2}{3}$;

(ii) $\frac{2}{3}<\mu<\frac{2}{1+\sqrt{2}}$ and either $p_{n}<p_{n}^{* *}$ or $p_{n}>p_{n}^{* *}$ and $n>n^{* *}$, where both $p_{n}^{* *}$ and $n^{* *}$ are finite and positive;

(iii) $\mu>\frac{2}{1+\sqrt{2}}, p_{n}>p_{n}^{*}$ and $n>n^{*}$, where both $p_{n}^{*}$ and $n^{*}$ are finite and positive; 2. The complete network is always pairwise stable.

Proposition 7 essentially shows that some amount of information sharing is consistent with equilibrium (point 2.), and is a feature of all equilibria when both signals' correlation and the number of agents are not too small (point 1.). This is in stark contrast with the traditional finding that no sharing of information occurs in Cournot oligopolies unless products are strongly differentiated (that is, unless strategies are either weak substitutes or complements - see the seminal works by Novshek and Sonnenschein (1982), Vives (1985), Kirby (1988), Li (1995)). To understand point 1., let us analyse the effects of the formation of one single link $i j$ on firms' information and behaviour in a Cournot oligopoly. Firms $i$ and $j$ get to observe each other signals and increase, as a result, the correlation of their equilibrium strategies. This has detrimental effects on their expected profits, which would outweigh the positive effect of a refined 
Fig. 1 Pairwise stable networks with four nodes
Complete Network

3-Node Component

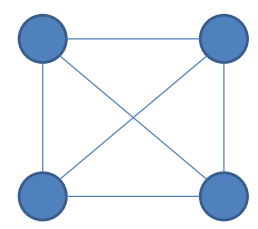

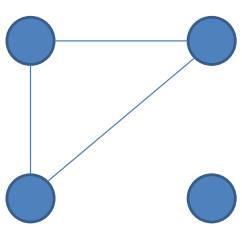

\section{Empty Network}
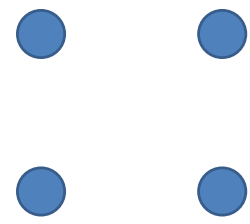

information if products were little differentiated and no other firms were in the market (this is the traditional conclusion for low levels of product differentiation). When $i$ and $j$ compete with other firms in the market, their exchange of information through the new link $i j$ provides both $i$ and $j$ with a refined prediction of the other firms' equilibrium behaviour. This refinement is due to signals' conditional correlation, and comes "at no cost", since it does not imply the transmission of $i$ 's and $j$ 's signal to the other firms in the market. The effect of such refinement on $i$ 's and $j$ 's expected profits is larger the larger the number of competitors, from which the requirement on $n$ in the proposition. Point 2. tells us that the failure of the empty network to be pairwise stable is not a symptom of a general instability problem, since a pairwise stable network (the complete network) exists for all parameters' ranges.

Understanding the effect of correlation in general is, however, hard. More correlation will in fact improve both the precision on unobserved signals before and after the additional link is formed. Intuitively, when an agent has little information, the gains (in terms of refined expectations) from one additional piece is substantial, leading to Point 1. of Proposition 7. However, as the stock of one's information builds up, the incentives to access more information may decrease. There may be therefore cases in which some information, but not all, is shared in equilibrium. The next four-player example fully characterise the set of pairwise stable networks, and provides some clearer insight on how correlation affects the incentives to share information in different networks (Fig. 1).

Example 4 Let $n=4, \mu=1$ and $p_{s}=1$. The pairwise stable networks in the various ranges of the correlation parameter $p_{n}$ are:

- $p_{n}<0.62$ : the complete network, the empty network and the network made of one complete component of 3 nodes and of one isolated node;

- $0.62<p_{n}<0.71$ : the complete and the empty networks.

- $0.71<p_{n}<0.75$ : the complete network, the empty network and the network made of one complete component of three nodes and of one isolated node; 
- $p_{n}>0.75$ : the complete network and the network made of one complete component of three nodes and of one isolated node;

For low levels of signals' correlation $\left(p_{n}<0.62\right)$, the architecture of pairwise stable networks is in accordance with our Proposition 3, dealing with the case of no correlation: fully connected components and, possibly, isolated nodes. Here the following incentives are at work: two players do not form a link when isolated, and form a link when they share the same degree. These incentives result in the empty and the complete network being stable. Moreover, players in the 3-node component have an incentive to form a link with the remaining isolated player who, in contrast, does not have such incentive. This is consistent with our discussion of Proposition 3, where we argued that the incentive for $i$ to link with $j$ increases with $i$ 's degree and decreases with $j$ 's degree. As correlation grows $\left(0.62<p_{n}<0.71\right)$, so do the incentives of the isolated player to gain information about the opponents' behaviour by forming one additional link, and this leads to the instability of the network containing the 3-node component. However, further increases of correlation $\left(0.71<p_{n}<0.75\right)$ decrease the incentive of each members of the 3-node component to link with the isolated node, whose behaviour is now predicted with high precision thanks to the high conditional correlation of signals. The isolated player remains thereby excluded from sharing. For large enough levels of correlation $\left(p_{n}>0.75\right)$, even single isolated players would form a link, and all pairwise stable networks display some amount of information sharing, consistently with point 1 . in Proposition 7 . Summing up, signals' correlation creates incentives to share information, but these incentives vanish when well connected (and informed) agents acquire a precise enough inference on unobserved signals.

We then turn to the $\mathrm{BC}$, where strategies are complements and payoffs also depend on the volatility of opponents' equilibrium strategies. We prove a partial counterpart of Proposition 5, showing that the universal sharing of information is always pairwise stable, and that all stable networks involve some amount of information sharing.

Proposition 8 In Keynes' Beauty Contest with strategic complements $(0<v<1)$ :

1. the complete network is pairwise stable;

2. the empty network is not pairwise stable.

Finally, we study the case in which also the covariance of equilibrium strategies and the state of the world affects payoffs. We focus on the public good game with linear benefits, for which we provide a full characterisation of pairwise stable networks. The intuition is similar to the one behind Corollary 2: both one's own equilibrium variance and opponents' covariance with the state of the world are beneficial, and both increase as a result of one additional link, resulting in the universal sharing of all information.

Proposition 9 In the public good game with linear benefits and quadratic costs the complete network is the unique pairwise stable architecture.

\section{Conclusions}

We have studied the incentives to bilaterally share information in linear quadratic games. Compared to the previous literature, our main contribution has been to frame 
the sharing problem in the general linear quadratic model, and to allow agents to share information by means of bilateral agreements rather than pooling all available information. We have focused on the ex-ante commitment to truthfully reveal information, and we have studied under which conditions agents make such commitments. As we have shown, the general linear quadratic formulation is rich enough to generate non trivial and incomplete architectures even in games with strategic complements, where incentives to share are strong. Our analysis has particularly focused on the role of signals' correlation in shaping incentives to share, and how and when incomplete network structure may arise in equilibrium. Our analysis of sharing has demanded a characterisation of the equilibrium use of information in networks, which extends previous work on Bayesian equilibrium in linear quadratic games to the case of networked information structures.

Acknowledgments We thank the associate editor and two anonymous referees for helpful suggestions and comments.

\section{Appendix 1}

Proof of Lemma 1 Assume $p_{n}=\gamma_{A}=\gamma_{A \theta}=0$. When passing from network $g$ to $g^{\prime}=g+i j$ condition (16) reduces to:

$$
-\gamma_{a} \cdot\left[\operatorname{var}\left(a_{i}^{g^{\prime}}\right)-\operatorname{var}\left(a_{i}^{g}\right)\right]>0 .
$$

Using now the expressions for variances:

$$
\operatorname{var}\left(a_{i}^{g^{\prime}}\right)-\operatorname{var}\left(a_{i}^{g}\right)=p_{s}\left[\sum_{h \in N_{i}^{g^{\prime}}}\left(\beta_{i h}^{g^{\prime}}\right)^{2}-\sum_{h \in N_{i}^{g}}\left(\beta_{i h}^{g}\right)^{2}\right]
$$

and the fact that from (18) all equilibrium coefficients applied to signal $y_{k}$ in $g$ and in $g^{\prime}$ only depend on the degree of $k$ in $g$ and $g^{\prime}$, respectively, we conclude that condition (16) is satisfied if and only if the following condition holds:

$$
\begin{aligned}
& \sum_{h \in N_{i}^{g^{\prime}}}\left(\beta_{i h}^{g^{\prime}}\right)^{2}-\sum_{h \in N_{i}^{g}}\left(\beta_{i h}^{g}\right)^{2}= \\
& -\gamma_{a}\left[\frac{1}{\left(2 \gamma_{a}+\gamma_{a A} n_{i}^{g}\right)^{2}}+\frac{1}{\left(2 \gamma_{a}+\gamma_{a A} n_{j}^{g}\right)^{2}}-\frac{1}{\left(2 \gamma_{a}+\gamma_{a A}\left(n_{i}^{g}-1\right)\right)^{2}}\right] \\
& \times\left[\frac{t \gamma_{a \theta}}{p_{s}}\right]^{2}>0 .
\end{aligned}
$$

Using now the definition of $\mu$, we then write:

$$
u_{i}(g+i j)>u_{i}(g) \Longleftrightarrow \frac{1}{\left(2+\mu\left(n_{i}^{g}-1\right)\right)^{2}}-\frac{1}{\left(2+\mu n_{i}^{g}\right)^{2}}<\frac{1}{\left(2+\mu n_{j}^{g}\right)^{2}}
$$


It is clear that, for any given $n_{i}^{g}$, if (21) is satisfied for a given $n_{j}^{g}=m$ then it remains true for all $n_{j}^{g}<m$. The function $f_{\mu}$, acting as a threshold for the formation of a new link $i j \notin g$, is then implicitly defined by the condition:

$$
\frac{1}{\left(2+\mu\left(n_{i}^{g}-1\right)\right)^{2}}-\frac{1}{\left(2+\mu n_{i}^{g}\right)^{2}}=\frac{1}{\left(2+\mu f_{\mu}\left(n_{i}^{g}\right)\right)^{2}} .
$$

Using similar steps we can define the function $F_{\mu}$, acting as a threshold for the severance of a link $i j \in g$. It is defined as the implicit solution of the following condition:

$$
\frac{1}{\left(2+\mu\left(n_{i}^{g}-2\right)\right)^{2}}-\frac{1}{\left(2+\mu\left(n_{i}^{g}-1\right)\right)^{2}}=\frac{1}{\left(2+\mu\left(F_{\mu}\left(n_{i}^{g}\right)-1\right)\right)^{2}} .
$$

We now turn to the proof of points (i)-(iv).

(i) The expression $\frac{1}{\left(2+\mu n_{i}^{g}\right)^{2}}$ is decreasing and convex in $n_{i}^{g}$. It follows that the LHS of (22) is decreasing in $n_{i}^{g}$, which in turns implies that the values of $n_{j}^{g}$ that satisfy (22) (that is, the value of $f_{\mu}\left(n_{i}^{g}\right)$ ) is increasing. Using condition (23), similar steps show that $F_{\mu}\left(n_{i}^{g}\right)$ is increasing in $n_{i}^{g}$.

(ii) We show that $f_{\mu}(m)>m$ for all $2 \leq m$. Let $n_{i}^{g}=f_{\mu}\left(n_{i}^{g}\right)=m$. From (22) $f_{\mu}(m)>m$ if and only if the following condition holds:

$$
\frac{1}{\left(2+\mu\left(n_{i}^{g}-1\right)\right)^{2}}-\frac{1}{\left(2+\mu n_{i}^{g}\right)^{2}}<\frac{1}{\left(2+\mu f_{\mu}\left(n_{i}^{g}\right)\right)^{2}} .
$$

or, equivalently,

$$
2(2+\mu(m-1))^{2}-(2+\mu m)^{2}>0
$$

It is directly verifiable that LHS of (25) is convex, the smaller root is negative for $0<\mu \leq 1$ and the larger root

$$
\frac{2(\mu-1)+\mu \sqrt{2}}{\mu}
$$

is smaller than 2 for $0<\mu \leq 1$. Therefore condition (24) is satisfied for all $m \geq 2$.

When $m=1$, condition (24) is satisfied if and only if $\mu<2(\sqrt{2}-1)<1$.

(iii) We now show that $f_{\mu}(m-1)=F_{\mu}(m)-1$. The value $f_{\mu}(m-1)$ solves the condition:

$$
\frac{1}{(2+\mu(m-2))^{2}}-\frac{1}{(2+\mu(m-1))^{2}}=\frac{1}{\left(2+\mu\left(f_{\mu}(m-1)\right)\right)^{2}} .
$$


The value $F_{\mu}(m)$ solves the condition:

$$
\frac{1}{(2+\mu(m-2))^{2}}-\frac{1}{(2+\mu(m-1))^{2}}=\frac{1}{\left(2+\mu\left(F_{\mu}(m)-1\right)\right)^{2}} .
$$

Comparison of conditions (26) and (27) directly implies the result.

(iv) Now we prove that $f_{\mu}(m)>F_{\mu}(m)$ for $m \geq 1$. First we claim that $f_{\mu}(m)-$ $f_{\mu}(m-1)>1$. From (22) we obtain:

$$
f_{\mu}(m)=\frac{2 \mu^{2}(\mu-4-2 n \mu)+\sqrt{\mu^{3}(2+(n-1) \mu)^{2}(2+n \mu)^{2}(4+(2 n-1) \mu)}}{\mu^{3}(4+(2 n-1) \mu)}
$$

Form (28) it is directly verifiable that $f_{\mu}(m)-f_{\mu}(m-1)>1$ for $m \geq 1$ and $0<\mu \leq 1$ (the complete proof is available upon request). This result, together with point (iii), imply point (iv).

Proof of Proposition 3 Point (1): If $\mu<0$ (the case of strategic complements), then by direct inspection of condition (21) we conclude that the link $i j$ will form (and will not be severed) for all levels of $n_{i}^{g}$ and of $n_{j}^{g}$. This implies that the complete network is the unique pairwise stable network.

Point (2). We first show that only regular networks can be pairwise stable when $\mu>0$. Assume $g$ is pairwise stable, and assume that $h$ is a non regular component of $g$. Consider now the node $i$ with maximal degree in $h$, and let $j$ be such that $n_{i}^{g}>n_{j}^{g}$ and $i j \in h$ (such link must exist for at least one node with maximal degree). This means that there exists some node $k \neq j$ such that $i k \in h$ and $j k \notin h$. By Lemma 1 , pairwise stability of $g$ imposes the following requirements on the degrees of nodes $i$, $j$ and $k$ :

$$
\begin{aligned}
n_{k}^{g} \leq F_{\mu}\left(n_{i}^{g}\right) \text { and } & n_{i}^{g} \leq F_{\mu}\left(n_{k}^{g}\right) \\
n_{j}^{g} & \leq F_{\mu}\left(n_{i}^{g}\right) \text { and } \quad n_{i}^{g} \leq F_{\mu}\left(n_{j}^{g}\right)
\end{aligned}
$$

Note now that since $n_{i}^{g}>n_{j}^{g}$ and since nodes' degrees are integers, then $n_{i}^{g}-1 \geq n_{j}^{g}$. This, together with (29), implies $n_{j}^{g} \leq F_{\mu}\left(n_{k}^{g}\right)-1$ and, together with point (iii) in Lemma 1, that $n_{j}^{g} \leq f\left(n_{k}^{g}-1\right)$. Finally, from point (i) in Lemma 1, we conclude that $n_{j}^{g}<f_{\mu}\left(n_{k}^{g}\right)$. This means that player $k$ has an incentive to form the link $j k$; stability of $g$ requires then that player $j$ has no incentive to form link $j k$, that is $n_{k}^{g}>f_{\mu}\left(n_{j}^{g}\right)$. This, together with $n_{k}^{g} \leq n_{i}^{g}$, implies that $n_{i}^{g}>f_{\mu}\left(n_{j}^{g}\right)$; using now(30) we obtain that $f_{\mu}\left(n_{j}^{g}\right)<F_{\mu}\left(n_{j}^{g}\right)$, contradicting point (iv) of Lemma 1.

Having shown that only regular components can belong to a stable network, the requirement that such components are fully connected comes from point (ii) in Lemma 1, where it shown that two agents with equal degree of at least 2 always have an incentive to form a link. 
The required ordering in the sizes $n\left(h_{1}\right), n\left(h_{2}\right), \ldots, n\left(h_{m}\right)$ comes from the observation that stability requires that no link is formed, and that a link joining two components does not form if and only if $n\left(h_{i}\right)>f_{\mu}\left(n\left(h_{i-1}\right)\right)$ for all $1 \leq k<m$.

Lastly, when $0<\mu<\frac{2}{1+\sqrt{2}}$ two isolated nodes would form a link (see point (ii) in Lemma 1), implying that two isolated nodes are incompatible with stability of the network.

Proof of Proposition 4 When $p_{n}=0$ and $\gamma_{A \theta}=0$, using expressions for variances, condition (16) simplifies as follows:

$$
\begin{aligned}
{\left[u_{i}^{e}\left(g^{\prime}\right)-u_{i}^{e}(g)\right]=} & \gamma_{A} p_{s}\left[\sum_{k \in N}\left(B_{i k}^{g^{\prime}}\right)^{2}-\sum_{k \in N}\left(B_{i k}^{g}\right)^{2}\right] \\
& -\gamma_{a} p_{s}\left[\sum_{h \in N_{i}^{g^{\prime}}}\left(\beta_{i h}^{g^{\prime}}\right)^{2}-\sum_{h \in N_{i}^{g}}\left(\beta_{i h}^{g}\right)^{2}\right]>0
\end{aligned}
$$

Consider now the networks $g$ and $g^{\prime}=g+i j$. Using the characterisation of equilibrium coefficients in (18), we note that $\beta_{i k}^{g^{\prime}}=\beta_{i k}^{g}$ for all $k \neq i, j$ and then $B_{i k}^{g^{\prime}}=B_{i k}^{g}$ for all $k \neq i, j$, since $n_{k}^{g^{\prime}}=n_{k}^{g}$ for all $k \neq i, j$. This implies that:

$$
\sum_{k \in N}\left(B_{i k}^{g^{\prime}}\right)^{2}-\sum_{k \in N}\left(B_{i k}^{g}\right)^{2}=\left(B_{i i}^{g^{\prime}}\right)^{2}-\left(B_{i i}^{g}\right)^{2}+\left(B_{i j}^{g^{\prime}}\right)^{2}-\left(B_{i j}^{g}\right)^{2}
$$

Also, using the fact that $n_{i}^{g^{\prime}}-1=n_{i}^{g}$ and $n_{j}^{g^{\prime}}-1=n_{j}^{g}$ from (18) we obtain:

$$
\begin{array}{r}
B_{i i}^{g^{\prime}}=-\frac{n_{i}^{g} \gamma_{a \theta} \frac{t}{p_{s}}}{2 \gamma_{a}+\gamma_{a A} n_{i}^{g}} \\
B_{i i}^{g}=-\frac{\left(n_{i}^{g}-1\right) \gamma_{a \theta} \frac{t}{p_{s}}}{2 \gamma_{a}+\gamma_{a A}\left(n_{i}^{g}-1\right)} \\
B_{i j}^{g^{\prime}}=-\frac{n_{j}^{g} \gamma_{a \theta} \frac{t}{p_{s}}}{2 \gamma_{a}+\gamma_{a A} n_{j}^{g}} \\
B_{i j}^{g}=-\frac{n_{j}^{g} \gamma_{a \theta} \frac{t}{p_{s}}}{2 \gamma_{a}+\gamma_{a A}\left(n_{j}^{g}-1\right)}
\end{array}
$$

Condition (31) can now be written as follows:

$$
\begin{aligned}
& {\left[u_{i}^{e}(g+i j)-u_{i}^{e}(g)\right]=} \\
& \quad=\gamma_{a \theta}^{2} \frac{t^{2}}{p_{s}} \gamma_{A}\left[\frac{\left(n_{i}^{g}\right)^{2}}{\left(2 \gamma_{a}+\gamma_{a A} n_{i}^{g}\right)^{2}}-\frac{\left(n_{i}^{g}-1\right)^{2}}{\left(2 \gamma_{a}+\gamma_{a A}\left(n_{i}^{g}-1\right)\right)^{2}}+\frac{\left(n_{j}^{g}\right)^{2}}{\left(2 \gamma_{a}+\gamma_{a A} n_{j}^{g}\right)^{2}}\right.
\end{aligned}
$$




$$
\begin{aligned}
& \left.-\frac{\left(n_{j}^{g}\right)^{2}}{\left(2 \gamma_{a}+\gamma_{a A}\left(n_{j}^{g}-1\right)\right)^{2}}\right]-\gamma_{a \theta}^{2} \frac{t^{2}}{p_{s}} \gamma_{a} \\
& \times\left[\frac{1}{\left(2 \gamma_{a}+\gamma_{a A} n_{i}^{g}\right)^{2}}+\frac{1}{\left(2 \gamma_{a}+\gamma_{a A} n_{j}^{g}\right)^{2}}-\frac{1}{\left(2 \gamma_{a}+\gamma_{a A}\left(n_{i}^{g}-1\right)\right)^{2}}\right]>0 .
\end{aligned}
$$

From direct inspection of (37) we see that both terms in squared brackets are positive, which, together with the assumptions that $\gamma_{a}<0$ and $\gamma_{A}>0$, implies that (37) is always satisfied and $i$ always has an incentive to form a link with $j$. So we can conclude that all links form.

Proof of Proposition 5 Agent $i$ has an incentive to link to $j$ iff expression (37) is strictly positive. In the $\mathrm{BC}$ we have the following restrictions: $\gamma_{a}=-1 ; \gamma_{A}=-\frac{(1-v)}{(n-1)^{2}}$; $\gamma_{a A}=\frac{2(1-v)}{n-1}$. Note first that for $n_{i}^{g}=n_{j}^{g}=1$ (37) is strictly positive iff:

$$
\frac{\left(n^{2}-2 n+v\right)\left(n^{2}-2+4 v-2 n v-v^{2}\right)}{\left(4(n-1)^{2}(n+v-2)^{2}\right)}>0 .
$$

We then compute the derivative of expression (37) with respect to $n_{i}^{g}$ for $n_{j}^{g}=1$ and show that is always positive. This implies that agent $i$ has an incentive to link to the singleton agent $j$, independently of $i$ 's degree. Then we compute the derivative of expression (37) with respect to $n_{j}^{g}$ for any arbitrary value of $n_{i}^{g}$ and we show that it is positive for any values of $n_{i}^{g}$ and $n_{j}^{g}$. This implies that (37) remains strictly positive for all $n_{i}^{g}<n$ and $n_{j}^{g}<n$, which implies the result. Exact computations of the derivatives involve long expressions and are available upon request.

Proof of Proposition 6 When $p_{n}=\gamma_{a A}=0$ and $g^{\prime}=g+i j$, using (18) and (33)-(36) we can rewrite condition (16) as follows:

$$
\begin{aligned}
\gamma_{a \theta}^{2} & \frac{t^{2}}{p_{S}} \gamma_{A} \\
& {\left[\frac{\left(n_{i}^{g}\right)^{2}}{2 \gamma_{a}}-\frac{\left(n_{i}^{g}-1\right)^{2}}{2 \gamma_{a}}\right]+\gamma_{a \theta} \frac{t^{2}}{p_{s}} \gamma_{A \theta}\left[-\frac{n_{i}^{g}}{2 \gamma_{a}}+\frac{n_{i}^{g}-1}{2 \gamma_{a}}\right] } \\
-\gamma_{a \theta}^{2} \frac{t^{2}}{p_{s}} \gamma_{a}\left[\frac{1}{2 \gamma_{a}}\right]>0 . &
\end{aligned}
$$

This is satisfied if and only if:

$$
\frac{\gamma_{a \theta} \gamma_{A}}{4 \gamma_{a}^{2}}\left(2 n_{i}^{g}-1\right)-\frac{\gamma_{A \theta}}{2 \gamma_{a}}-\frac{\gamma_{a \theta}}{4 \gamma_{a}}>0
$$

where we have used the assumption that $\gamma_{a \theta}>0$. Condition (40) can be written as:

$$
\frac{\gamma_{A}}{\gamma_{a}}\left(2 n_{i}^{g}-1\right)<\frac{2 \gamma_{A \theta}+\gamma_{a \theta}}{\gamma_{a \theta}} .
$$


Case $\gamma_{A}=0$. When $2 \gamma_{A \theta}+\gamma_{a \theta}>0$, (41) is always satisfied and the complete network is the unique pairwise stable network. When $2 \gamma_{A \theta}+\gamma_{a \theta}<0$, (41) is never satisfied and the empty network is the unique pairwise stable network.

Case $\gamma_{A}>0$. When $2 \gamma_{A \theta}+\gamma_{a \theta}>0$, (41) is always satisfied. When $2 \gamma_{A \theta}+\gamma_{a \theta}<0$, (41) is satisfied for $n_{i}^{g}>\frac{1}{2}\left(\frac{2 \gamma_{A \theta}+\gamma_{a \theta}}{\gamma_{a \theta}} \frac{\gamma_{a}}{\gamma_{A}}+1\right) \equiv m>0$. In this case the empty network is trivially stable, as is the complete network. The only other stable architecture is such that all nodes with positive degree have degree larger than $m$, and all such nodes are linked to each other.

Case $\gamma_{A}<0$. Condition (41) is never satisfied when $2 \gamma_{A \theta}+\gamma_{a \theta}<0$ (from which the empty network is the unique pairwise stable network), while if $2 \gamma_{A \theta}+\gamma_{a \theta}>0$ the relevant condition for agent $i$ to form a link is the following:

$$
n_{i}^{g}<\frac{1}{2}\left(\frac{2 \gamma_{A \theta}+\gamma_{a \theta}}{\gamma_{a \theta}} \frac{\gamma_{a}}{\gamma_{A}}+1\right) \equiv m>0
$$

Here, only agents with a low enough degree would form a link. If the threshold degree $m$ is less than zero, then the empty network is the unique pairwise stable network; if not, no pairwise stable network exists. To see this, note that two nodes who are linked in a stable network must have degree less than $m$. But in this case they wish to form a link to the agents to which they are not linked. If these agents have degree less than $m$ they also want to link, then we contradict the stability of the network. If they do not wish to link, then they must have a degree which is larger than $m$, in which case they wish to sever a link. Finally, the empty network is not stable since two nodes of degree zero wish to form a link.

Proof of Proposition 7 Proof of point 1: The proof is organised in several steps, and goes by studying the difference in expected profits of two firms, 1 and 2, in the complete networks $g^{c}$ and in the network $g^{-12} \equiv\left\{g^{c}-12\right\}$. We first compute equilibrium strategies in $g^{c}$. The updating coefficient in $g^{c}$ is for every $i$ :

$$
k_{1}^{i g^{c}}=\frac{t}{p_{s}+(n-1) p_{n}} .
$$

We obtain the following common equilibrium coefficient:

$$
\beta^{g^{c}}=-\frac{t \gamma_{a \theta}}{\left(p_{s}+(n-1) p_{n}\right)\left(2 \gamma_{a}+\gamma_{a A}(n-1)\right)}
$$

For $g^{-12} \equiv\left\{g^{c}-12\right\}$, the updating coefficients are:

$$
\begin{aligned}
& k_{1}^{i g^{-12}}=\frac{t}{p_{s}+(n-2) p_{n}}, \quad k_{2}^{i}=\frac{p_{n}}{p_{s}+(n-2) p_{n}}, \quad i=1,2 \\
& k_{1}^{i g^{-12}}=\frac{t}{p_{s}+(n-1) p_{n}}, \quad \forall i \geq 3
\end{aligned}
$$


We obtain the following equilibrium coefficients for firms 1 and 2:

$$
\begin{aligned}
\beta_{11}^{g^{-12}} & =\beta_{22}^{g^{-12}}=-\frac{t \gamma_{a \theta}}{2 \gamma_{a}\left(p_{s}+(n-2) p_{n}\right)+\gamma_{a A}\left((n-2) p_{s}+(5+n(n-4)) p_{n}\right)} \\
\beta_{1 j}^{g^{-12}} & =\beta_{2 j}^{g^{-12}} \\
& =-\frac{t\left(2 \gamma_{a}+(n-2) \gamma_{a A}\right) \gamma_{a \theta}}{\left(2 \gamma_{a}+(n-1) \gamma_{a A}\right)\left(2\left(p_{s}+(n-2) p_{n}\right) \gamma_{a}+\gamma_{a A}\left((n-2) p_{s}+(5+n(n-4)) p_{n}\right)\right)}, \quad \forall j \geq 3
\end{aligned}
$$

From (16), we can express the difference $u^{e}\left(g^{c}\right)-u^{e}\left(g^{-12}\right)$ in the expected profits of firm 1 (and, by symmetry, of firm 2) in $g^{c}$ and in $g^{-12}$ as proportional to:

$$
\begin{aligned}
& n \cdot\left(\beta_{i j}^{g^{c}}\right)^{2}\left(p_{s}+(n-1) p_{n}\right)-\left(\left(\beta_{11}^{g^{-12}}\right)^{2}+(n-2)\left(\beta_{1 j}^{g^{-12}}\right)^{2}\right) p_{s}- \\
& \quad-(n-2) \beta_{1 j}^{g^{-12}}\left(2 \beta_{11}^{g^{-12}}+(n-3) \beta_{1 j}^{g^{-12}}\right) p_{n} .
\end{aligned}
$$

Plugging in the values of the $\beta$ coefficients, we obtain the following expression:

$$
\frac{\left(p_{s}-p_{n}\right) t^{2}\left(4\left(p_{s}+(n-2) p_{n}\right)(1+(n-3) \mu)+\left((7+(n-6) n) p_{s}+(n(19+(n-8) n)-16) p_{n}\right) \mu^{2}\right) \gamma_{a \theta}^{2}}{\left(p_{s}+(n-1) p_{n}\right)(2+(n-1) \mu)^{2}\left(2 p_{s}+2(n-2) p_{n}+(n-2) p_{s} \mu+(5+(n-4) n) p_{n} \mu\right)^{2} \gamma_{a}^{2}}
$$

The denominator of the above equation is always strictly positive for all admissible values of the parameters; moreover the sign is the same as the sign of the following expression:

$$
\begin{aligned}
& 4\left(p_{s}+(n-2) p_{n}\right)(1+(n-3) \mu)+ \\
& \quad+\left((7+(n-6) n) p_{s}+(n(19+(n-8) n)-16) p_{n}\right) \mu^{2}
\end{aligned}
$$

We divide it in two terms. The first, $4\left(p_{s}+(n-2) p_{n}\right)(1+(n-3) \mu)$, is always positive: indeed by assumption $\mu>0$ and the proof follows directly; it can be directly verified that the second term is positive for $n \geq 5$. Therefore (46) could be negative only for $n=3$ and $n=4$. But for $n=3$ (46) becomes $4\left(p_{s}+p_{n}\right)-2\left(p_{s}+2 p_{n}\right) \mu^{2}$ and for $n=4$ (46) becomes $4\left(p_{s}+2 p_{n}\right)(1+\mu)-\left(p_{s}+4 p_{n}\right) \mu^{2}$ and, by the assumption that $0<\mu<1$, both terms are strictly positive.

Proof of Point 2 We study the difference in expected profits of two agents, 1 and 2, in the empty network $g^{\varnothing}$ and in the network $g^{12} \equiv\{12\}$. The updating coefficients for $g^{\varnothing}$ are:

$$
k_{1}^{i g^{\varnothing}}=\frac{t}{p_{s}}, \quad k_{2}^{i g^{\varnothing}}=\frac{p_{n}}{p_{s}}, \quad \forall i
$$

from which we obtain the common coefficient of agents' equilibrium strategies:

$$
\beta_{i i}^{g^{\varnothing}}=-\frac{\gamma_{a \theta} t}{2 \gamma_{a} p_{s}+\gamma_{a A}(n-1) p_{n}}, \quad \forall i
$$


The updating coefficients for $g^{12} \equiv\{12\}$ are:

$$
\begin{aligned}
& k_{1}^{i g^{12}}=\frac{t}{p_{s}+p_{n}} \text { for } i=1,2 \\
& k_{1}^{i g^{12}}=\frac{t}{p_{s}} \text { for all } i \geq 3 \\
& k_{2}^{i g^{12}}=\frac{p_{n}}{p_{s}+p_{n}} \text { for } i=1,2 \\
& k_{2}^{i g^{12}}=\frac{p_{n}}{p_{s}} \text { for } i \geq 3
\end{aligned}
$$

We obtain the following equilibrium coefficients for agents 1 and 2:

$$
\begin{aligned}
\beta_{11}^{g^{12}} & =\beta_{12}^{g^{12}}=\beta_{21}^{g^{12}}=\beta_{22}^{g^{12}} \\
& =-\frac{t\left(2 p_{s} \gamma_{a}-\gamma_{a A} p_{n}\right) \gamma_{a \theta}}{4 p_{s}\left(p_{s}+p_{n}\right) \gamma_{a}^{2}+2\left(p_{s}+p_{n}\right)\left(p_{s}+(n-3) p_{n}\right) \gamma_{a} \gamma_{a A}+p_{n}\left((n-3) p_{s}-(3 n-5) p_{n}\right) \gamma_{a A}^{2}}
\end{aligned}
$$

From (16), we can express the difference in profits of agent 1 (and, by symmetry, of agent 2) in $g^{\varnothing}$ and in $g^{12}$ as:

$$
\left(\beta_{i i}^{g^{\varnothing}}\right)^{2} p_{s}-2\left(\beta_{11}^{g^{12}}\right)^{2}\left(p_{s}+p_{n}\right) .
$$

Plugging in (48) the values of the $\beta$ coefficients, recalling the definition of $\mu$ and letting $p \equiv p_{s}+p_{n}$ we obtain the following expression:

$$
\begin{aligned}
& \frac{t^{2} \gamma_{a \theta}^{2}}{\gamma_{a}^{2}}\left[\frac{p_{s}}{\left(2 p_{s}+(n-1) p_{n} \mu\right)^{2}}-\right. \\
& \left.\frac{2 p\left(p_{n} \mu-2 p_{s}\right)^{2}}{\left(4 p_{s} p+2 p\left(p_{s}+(n-3) p_{n}\right) \mu-p_{n}\left((3 n-5) p_{n}-(n-3) p_{s}\right) \mu^{2}\right)^{2}}\right]
\end{aligned}
$$

It can be shown that the denominator of the above expression is strictly positive. Its sign of is therefore the sign of the numerator of the above expression, which can be written in the following form:

$$
a \cdot n^{2}+b \cdot n+c
$$

where

$$
\begin{aligned}
a= & \left(p_{s}-p_{n}\right) p_{n}^{2} \mu^{2}\left(4 p_{s} p(\mu-1)+\left(p_{s}^{2}-5 p_{s} p_{n}+2 p_{n}^{2}\right) \mu^{2}\right) \\
b= & 2\left(p_{s}-p_{n}\right) p_{n} \mu \cdots\left(-8 p_{s}^{2} p+4 p_{s} p\left(2 p_{s}+3 p_{n}\right) \mu\right. \\
& \left.+2 p_{s}\left(p_{s}-8 p_{n}\right) p \mu^{2}-p_{n}\left(3 p_{s}^{2}-11 p_{s} p_{s}+2 p_{n}^{2}\right) \mu^{3}\right)
\end{aligned}
$$




$$
\begin{aligned}
c= & \left(p_{s}-p_{n}\right)\left[2 p_{n}^{4} \mu^{4}+p_{s} p_{n}^{3} \mu^{2}((44-21 \mu) \mu-36)+4 p_{s}^{4}(\mu(4+\mu)-4)\right. \\
& \left.-4 p_{s}^{3} p_{n}(\mu-1)(3 \mu(4+\mu)-4)+p_{s}^{2} p_{n}^{2} \mu(48+\mu(\mu(32+9 \mu)-76))\right]
\end{aligned}
$$

The proof continues now by studying the sign of (49).

We first note that the roots $\left(n_{-}, n_{+}\right)$of (49) are real (since $\left.b^{2}-4 a c \geq 0\right)$, distinct and finite as long as $a \neq 0$. With this in mind, we now look for conditions under which expression (49) is concave. Such conditions will tell us whether the sign of (49) becomes negative for $n$ large enough.

Lemma 2 If $\mu<\frac{2}{1+\sqrt{2}}$ then (49) is concave. If $\mu>\frac{2}{1+\sqrt{2}}$ then there exists $p_{n}^{*}$ such that for all $p_{n}>p_{n}^{*}(49)$ is concave, otherwise it is convex.

Proof of Lemma 2 Note that concavity of (49) depends on the sign of term $a$ in (49). This term is negative for $\mu<0$. Moreover, the sign of $a$ is the sign of the following term:

$$
\left(4 p_{s} p(\mu-1)+\left(p_{s}^{2}-5 p_{s} p_{n}+2 p_{n}^{2}\right) \mu^{2}\right)
$$

Let us evaluate the roots of (50) as a function of $p_{n}$. We find:

$$
\frac{4 p_{s}(1-\mu)+5 p_{s} \mu^{2} \pm p_{s}(\mu-2) \sqrt{4(1-\mu)+17 \mu^{2}}}{4 \mu^{2}}
$$

Since the largest root yields a value which exceeds $p_{s}$, we only consider the smaller root denoted by $p_{n}^{*}$. Note here that the second derivative of (50) with respect to $p_{n}$ is positive (so that $a$ is a convex function of $p_{n}$ ). This directly implies that $a$ is negative for all $p_{n}>p_{n}^{*}$. We then turn to the analysis of the root $p_{n}^{*}$ in relation to the parameter $\mu$. We show that if $\mu<\frac{2}{1+\sqrt{2}}$ then $p_{n}^{*}<0$, implying that $a<0$ for all parameters' values; moreover, when $\mu>\frac{2}{1+\sqrt{2}}$, we show that $p_{n}^{*}>0$ and that $p_{n}^{*}$ is increasing in $\mu$. In this latter case, $a<0$ for all values $p_{n}^{*}<p_{n}<p_{s}$.

Consider again the smaller root in (51):

$$
p_{n}^{*}=\frac{4 p_{s}(1-\mu)+5 p_{s} \mu^{2}+p_{s}(\mu-2) \sqrt{4(1-\mu)+17 \mu^{2}}}{4 \mu^{2}} .
$$

Expression (52) is null for the following values of $\mu$ :

$$
\mu_{-}=2(-1-\sqrt{2}) ; \mu_{+}=\frac{2}{1+\sqrt{2}}
$$

Moreover, the expression (52) is strictly increasing in $\mu$ for all values of $\mu$ in the range $(0,1]$. This implies that $p_{n}^{*}<0$ for all $0<\mu<\mu_{+}$, and that $p_{s}>p_{n}^{*}>0$ for all $\mu_{+}<\mu \leq 1$.

Having established conditions under which (49) is concave in $n$, we study its sign by establishing a few facts about the behaviour of (49) at the point $n=2$. 
Lemma 3 At $n=2$ : i) expression (49) is negative for $\mu<\frac{2}{3}$, is positive for $\mu>\frac{2}{1+\sqrt{2}}$ and in the intermediate range is positive if and only if $p_{n}>\frac{p_{s}\left(4-4 \mu-\mu^{2}\right)}{2 \mu^{2}} \equiv p_{n}^{* *}$. ii) Moreover, there exists $\hat{p}_{n}>0$ such that (49) is increasing in $n$ if $p_{n}<\hat{p}_{n}$ and $\mu>\frac{2}{1+\sqrt{2}}$, otherwise (49) is decreasing in $n$.

Proof of Lemma 3 Point (i) follows from direct computation, and is consistent with Proposition 4.4 in Raith (1996) for the specific case of Cournot oligopoly, setting $n=2$. Point (ii) is proved as follows. The first derivative of (49) at $n=2$ is given by:

$$
2\left(p_{s}-p_{n}\right) p_{n} p \mu\left(2 p_{s}-p_{n} \mu\right)\left(p_{s}(\mu(4+\mu)-4)-2 p_{n} \mu^{2}\right) .
$$

The sign of (54) is the same as the sign of the following expression:

$$
\mu\left(p_{s}(\mu(4+\mu)-4)-2 p_{n} \mu^{2}\right)
$$

The expression in brackets in (55) is positive for $p_{n}<\frac{p_{s}(\mu(4+\mu)-4)}{2 \mu^{2}} \equiv \hat{p}_{n}$. It is directly verifiable that $\hat{p}_{n}$ is negative for $\mu<\frac{2}{1+\sqrt{2}}$ and positive for $\mu>\frac{2}{1+\sqrt{2}}$.

We are now ready to prove Proposition 7.

Point (i) $\left(\mu<\frac{2}{3}\right)$. we know from Lemma 3 that at $n=2$ (49) is negative and decreasing in $n$, and from Lemma 2 we know that (49) is concave in $n$. This two facts tell us the all points $n \geq 2$ are in the right (and decreasing) branch of the parabola (49). We conclude that (49) is negative for all $n \geq 2$.

Point (ii) $\left(\frac{2}{3}<\mu<\frac{2}{1+\sqrt{2}}\right)$. From Lemma 2 and Lemma 3 we know that (49) is concave and decreasing in $n$ at $n=2$. These two facts imply that all points $n \geq 2$ are in the right (and decreasing) branch of the parabola (49). In this range of values for $\mu$, however, (49) can be either positive or negative at $n=2$, depending on the value of $p_{n}$ (see Lemma 3 point (i)). Suppose first that (49) is negative at $n=2$; in this case, the two real roots of (49) are strictly smaller than 2 , and (49) remains negative for all $n \geq 2$. Suppose then that (49) is positive at $n=2$; in this case, the larger real root $n_{+}$ must be larger than 2, so that (49) is negative for all $n>n_{+}$.

Point (iii) $\left(\mu>\frac{2}{1+\sqrt{2}}\right)$. In this range, (49) is concave in $n$ if and only if if $p_{n}>p_{n}^{*}>0$, otherwise it is convex (Lemma 2). Moreover, we know from Lemma 3 that at $n=2$ (49) is positive. Consider first the case $p_{n}>p_{n}^{*}((49)$ concave). Here, the larger real root $n_{+}$must be larger than 2, so that for all $n>n_{+}$(49) is negative. Consider then the case $p_{n}<p_{n}^{*}((49)$ convex). Here, at $n=2$ (49) is increasing in $n$ if $p_{n}<\hat{p}_{n}$. Since $\hat{p}_{n}=p_{n}^{*}$ for $\mu=\frac{2}{1+\sqrt{2}}$ and for $\mu>\frac{2}{1+\sqrt{2}}$ the difference $\hat{p}_{n}-p_{n}^{*}$ is increasing in $\mu^{2}$, it follows that $p_{n}^{*}<\hat{p}_{n}$ for all $\mu>\frac{2}{1+\sqrt{2}}$ and that (49) is increasing in $n$ at $n=2$. Since in this case (49) is convex, we conclude that the two real roots $\left(n_{-}, n_{+}\right)$are smaller than 2 , and that (49) is positive for $n \geq 2$.

\footnotetext{
2 More precisely, the first derivative of the expression $\frac{p_{s}(\mu(4+\mu)-4)}{2 \mu^{2}}-p_{n}$ is increasing in $\mu$.
} 
Proof of Proposition 8 We replace the parameters in (1) with those specific for the BC: $\gamma_{a}=-1 ; \gamma_{\theta}=-v ; \gamma_{a \theta}=2 v ; \gamma_{A}=-\frac{(1-v)}{(n-1)^{2}} ; \gamma_{a A}=\frac{2(1-v)}{n-1}$.

Point 1. We study again the difference in expected profits of two firms, 1 and 2, in the complete networks $g^{c}$ and in the network $g^{-12} \equiv\left\{g^{c}-12\right\}$. The updating coefficient in $g^{c}$ is:

$$
k_{1}^{i g^{c}}=\frac{t}{p_{s}+(n-1) p_{n}},
$$

from which we obtain the following common equilibrium coefficient:

$$
\beta^{g^{c}}=-\frac{t}{\left(p_{s}+(n-1) p_{n}\right)} .
$$

For $g^{-12} \equiv\left\{g^{c}-12\right\}$, the updating coefficients are:

$$
\begin{aligned}
& k_{1}^{i g^{-12}}=\frac{t}{p_{s}+(n-2) p_{n}}, \quad k_{2}^{i}=\frac{p_{n}}{p_{s}+(n-2) p_{n}}, \quad i=1,2 \\
& k_{1}^{i g^{-12}}=\frac{t}{p_{s}+(n-1) p_{n}}, \quad \forall i \geq 3
\end{aligned}
$$

We obtain the following equilibrium coefficients for firms 1 and 2 and for firms $j, k>2$ :

$$
\begin{aligned}
\beta_{11}^{g^{-12}} & =\beta_{22}^{g^{-12}}=-\frac{t(n-1) v}{p_{s}+(n-2) p_{s} v+p_{n}(n-3+(5+(n-4) n) v)} \\
\beta_{1 j}^{g^{-12}} & =\beta_{2 j}^{g^{-12}} \\
& =-\frac{t+(n-2) t v}{p_{s}+(n-2) p_{s} v+p_{n}(n-3+(5+(n-4) n) v)}, \\
\beta_{j 1}^{g^{-12}} & =\beta_{j 2}^{g^{-12}} \\
& =-\frac{(n-1)\left((n-2) p_{n}+p_{s}\right) t v}{\left((n-1) p_{n}+p_{s}\right)\left(p_{s}+(n-2) p_{s} v+p_{n}(n-3+(5+(n-4) n) v)\right)} \\
\beta_{j j}^{g^{-12}} & =\beta_{j k}^{g^{-12}} \\
& =-\frac{t\left(p_{s}+(n-2) p_{s} v+(n-1) p_{n}(1+(n-3) v)\right)}{\left((n-1) p_{n}+p_{s}\right)\left(p_{s}+(n-2) p_{s} v+p_{n}(n-3+(5+(n-4) n) v)\right)}
\end{aligned}
$$

We will now write down the change in expected payoff of agent 1 moving from $g^{c}$ to $g^{-12}$ following condition (17-19) and (21). The terms used to compute the other players' aggregate volatility are given by:

$$
\begin{aligned}
B_{1 k}^{g^{c}} & =(n-1) \beta_{11}^{g^{c}} ; \\
B_{11}^{g^{-12}} & =(n-2) \beta_{31}^{g^{-12}}
\end{aligned}
$$




$$
\begin{aligned}
& B_{12}^{g^{-12}}=\beta_{11}^{g^{-12}}+(n-2) \beta_{31}^{g^{-12}} ; \\
& B_{1 k}^{g^{-12}}=\beta_{13}^{g^{-12}}+(n-2) \beta_{33}^{g^{-12}} .
\end{aligned}
$$

From (21) we express the change in payoff moving from $g^{-12}$ to $g^{c}$ as follows:

$$
\begin{aligned}
& -\gamma_{a}\left[\left(p_{s} n+n(n-1) p_{n}\right)\left(\beta_{11}^{g^{c}}\right)^{2}-p_{s}\left(\beta_{11}^{g^{-12}}\right)^{2}-(n-2)\left(\beta_{1 k}^{g^{-12}}\right)^{2} p_{s}-\left(2(n-2) \beta_{11}^{g^{-12}}\right.\right. \\
& \left.\left.\beta_{1 k}^{g^{-12}}+(n-2)(n-3)\left(\beta_{1 k}^{g^{-12}}\right)^{2}\right) p_{n}\right]+\gamma_{A}\left[n p_{s}\left(B_{21}^{g^{c}}\right)^{2}+n(n-1)\left(B_{i k}^{g^{c}}\right)^{2} p_{n}\right. \\
& -p_{s}\left(\left(B_{12}^{g^{-12}}\right)^{2}+\left(B_{11}^{g^{-12}}\right)^{2}+(n-2)\left(B_{13}^{g^{-12}}\right)^{2}\right)-2 p_{n}\left(\left(B _ { 1 1 } ^ { g ^ { - 1 2 } } \left(B_{12}^{g^{-12}}\right.\right.\right. \\
& \left.\left.\left.\quad+(n-2) B_{13}^{g^{-12}}\right)+B_{12}^{g^{-12}}(n-2) B_{13}^{g^{-12}}+\left(B_{13}^{g^{-12}}\right)^{2}(n-2)(n-3) / 2\right)\right]
\end{aligned}
$$

Now we show that this expression is never negative for all values of $v, p_{n}$ and $p_{s}$ in the ranges $0<v \leq 1$ and $p_{n}<p_{s}$. Replacing the coefficients we get the following expression:

$$
\frac{\left(p_{s}-p_{n}\right) t^{2} v\left(p_{n}\left(2 n+12 v-6+n \cdot v(n(n-2)-4)+v^{2}(n(7-2 n)-8)\right)+p_{s}\left(2+v\left(n^{2}-4+3 v-2 n v\right)\right)\right)}{\left((n-1) p_{n}+p_{s}\right)\left(p_{s}+(n-2) p_{s} v+p_{n}(n-3+(5+(n-4) n) v)\right)^{2}}
$$

It can be shown that the denominator of (62) is strictly positive. Then the sign of (62) is therefore the sign of its numerator, which can be written in the following form:

$$
a \cdot v^{2}+b \cdot v+c
$$

where

$$
\begin{gathered}
a=p_{n}(n(7-2 n)-8)+p_{s}(3-2 n) \\
b=12 p_{n}+n \cdot p_{n}(n(n-2)-4)+p_{s}\left(n^{2}-4\right) \\
c=2 p_{s}+p_{n}(2 n-6)
\end{gathered}
$$

The proof continues now by studying the sign of (63).

We first note that the roots $\left(n_{-}, n_{+}\right)$of $(63)$ are real (since $\left.b^{2}-4 a c \geq 0\right)$, distinct and finite (since $a \neq 0$ ). Moreover by a direct inspection of (63) we see that is concave and that its smaller root is negative and the larger one is greater than 1 . Then (62) is positive for all parameter values, implying that the complete network is always stable.

Point 2: We study the difference in expected profits of two agents, 1 and 2, in the empty network $g^{\varnothing}$ and in the network $g^{12} \equiv\{12\}$. The updating coefficients for $g^{\varnothing}$ are:

$$
k_{1}^{i g^{\varnothing}}=\frac{t}{p_{s}}, \quad k_{2}^{i g^{\varnothing}}=\frac{p_{n}}{p_{s}}, \quad \forall i
$$

from which we obtain the common coefficient of agents' equilibrium strategies:

$$
\beta^{g^{\varnothing}}=-\frac{t v}{p s+p n(v-1)} .
$$


and

$$
\begin{gathered}
B_{i i}^{g^{\varnothing}}=0 \\
B_{i j}^{g^{\varnothing}}=\beta^{g^{\varnothing}} \text { for } i \neq j
\end{gathered}
$$

The updating coefficients for $g^{12} \equiv\{12\}$ are:

$$
\begin{aligned}
& k_{1}^{i g^{12}}=\frac{t}{p_{s}+p_{n}} \quad \text { for } i=1,2 \\
& k_{1}^{i g^{12}}=\frac{t}{p_{s}} \quad \text { for all } i \geq 3 \\
& k_{2}^{i g^{12}}=\frac{p_{n}}{p_{s}+p_{n}} \quad \text { for } i=1,2 \\
& k_{2}^{i g^{12}}=\frac{p_{n}}{p_{s}} \text { for } i \geq 3
\end{aligned}
$$

We obtain the following equilibrium coefficients:

$$
\begin{aligned}
& \beta_{11}^{g^{12}}=\beta_{12}^{g^{12}}=\beta_{21}^{g^{12}}=\beta_{22}^{g^{12}} \\
& =-\frac{(n-1) t v\left(p_{n}(1-v)+(n-1) p_{s}\right)}{(n-1) p_{s}^{2}(n+v-2)+p_{n}^{2}(v-1)(n(n-1-3 v)+5 v-2)+p_{n} p_{s}(n-2+v)(2+(n-3) v)} \\
& \beta_{k k}^{g^{12}}=\frac{(n-1) t v\left(p_{n}(2+n-3 v)+p_{s}(n-2+v)\right)}{(n-1) p_{s}^{2}(n-2+v)+p_{n}^{2}(v-1)(n-2(n-1-3 v)+5 v)+p_{n} p_{s}(n-2+v)(2+(n-3) v)},
\end{aligned}
$$

and:

$$
\begin{aligned}
& B_{11}^{g^{12}}=\beta_{21}^{g^{12}} \\
& B_{1 k}^{g^{12}}=\beta_{k k}^{g^{12}} .
\end{aligned}
$$

Using (16) we can express the difference in profits of agent 1 (and, by symmetry, of agent 2) in $g^{\varnothing}$ and in $g^{12}$ as:

$$
\begin{aligned}
& -\gamma_{a}\left[\left(\beta_{i i}^{g^{\varnothing}}\right)^{2} p_{s}-2\left(\beta_{11}^{g^{12}}\right)^{2}\left(p_{s}+p_{n}\right)\right]+\gamma_{A}\left[(n-1) B_{i j}^{g^{\varnothing} 2} p_{s}\right. \\
& +(n-1)(n-2) B_{i j}^{g^{\varnothing} 2} p_{n}-\left(2\left(B_{11}^{g^{12}}\right)^{2}+(n-2)\left(B_{1 k}^{g^{12}}\right)^{2}\right) p_{s} \\
& \left.\quad-\left(2\left(B_{11}^{g^{12}}\right)^{2}+(4 n-8) B_{11}^{g^{12}} B_{1 k}^{g^{12}}+(n-2)(n-3)\left(B_{1 k}^{g^{12}}\right)^{2}\right) p_{n}\right]
\end{aligned}
$$

The proof then goes through the following steps (complete proofs from authors upon request): we plug in the coefficients' expressions, so we get an expression in $n$, $p_{s}, p_{n}$ and $v$. Then we find that: i) (69) is strictly positive for $p_{n}=0$ for all parameters' values; ii) (69) is equal zero for $p_{n}=p_{s}$ for all parameters' values; iii) the derivative of (69) respect to $p_{n}$ computed in $p_{n}=p_{s}$ is strictly negative for all parameters' values; iv) (69) never is negative for $p_{n} \in\left(0, p_{s}\right)$ and for all other parameters' value. 
All these evidences are enough to say that (69) is positive for all parameters' values and, consequently, the empty network is not pairwise stable.

Proof of Proposition 9 We replace the parameters in (1) with those specific for the Public Good Game: $\gamma_{a}=-b ; \gamma_{A \theta}=1 ; \gamma_{a \theta}=1$. Expression (6) becomes:

$$
\beta_{i h}^{g}=\frac{t}{2 b\left(p_{s}+\left(n_{i}^{g}-1\right) p_{n}\right)}, \quad \forall h \in N_{i}^{g}
$$

The incentives to form a new link are given by (16) which, when $\left(g^{\prime}=g+i j\right)$, is rewritten as follows:

$$
\left[\operatorname{cov}\left(A_{i}^{g^{\prime}}, \theta\right)-\operatorname{cov}\left(A_{i}^{g}, \theta\right)\right]+b \cdot\left[\operatorname{var}\left(a_{i}^{g^{\prime}}\right)-\operatorname{var}\left(a_{i}^{g}\right)\right]
$$

Note that the term inside the first brackets depends only on the covariance of the action of agent $j$ and $\theta$. Therefore using (70) and (18), and noting that passing from $g$ to $g^{\prime}$ only agents $i$ 's and $j$ 's coefficients change, we can write (71) as:

$$
\frac{t^{2}}{2 b}\left[\frac{n_{j}+1}{\left(p_{s}+n_{j} p_{n}\right)}-\frac{n_{j}}{\left(p_{s}+\left(n_{j}-1\right) p_{n}\right)}\right]+\frac{t^{2}}{4 b} \cdot\left[\frac{n_{i}+1}{p_{s}+n_{i} p_{n}}-\frac{n_{i}}{p_{s}+\left(n_{i}-1\right) p_{n}}\right] .
$$

Direct inspection of (72) show that (72) is strictly positive as long as $p_{s}>p_{n}$. Therefore every incomplete network is not stable, resulting in the complete network being the unique pairwise stable architecture.

\section{Appendix 2}

Equilibrium coefficients for the star network solve the following system of equations (we have used symmetry where possible):

$$
\begin{gathered}
\beta_{i i}^{g^{s}}=-\frac{1}{2 \gamma_{a}}\left(\gamma_{A \theta} k_{1}^{i g^{s}}+\gamma_{a A} 3 \beta_{i h}^{g^{s}}\right) \\
\beta_{i h}^{g^{s}}=-\frac{1}{2 \gamma_{a}}\left(\gamma_{A \theta} k_{1}^{i g^{s}}+\gamma_{a A} \beta_{h h}^{g^{s}}\right) \\
\beta_{h h}^{g^{s}}=-\frac{1}{2 \gamma_{a}}\left(\gamma_{A \theta} k_{1}^{h g^{s}}+\gamma_{a A}\left(\beta_{i h}^{g^{s}}\left(1+2 k_{2}^{h g^{s}}\right)+2 k_{2}^{h g^{s}} \beta_{h h}^{g^{s}}\right)\right) \\
\beta_{h i}^{g^{s}}=-\frac{1}{2 \gamma_{a}}\left(\gamma_{A \theta} k_{1}^{h g^{s}}+\gamma_{a A}\left(\beta_{i i}^{g^{s}}+\beta_{i h}^{g^{s}} 2 k_{2}^{h g^{s}}+2 \beta_{h i}^{g^{s}}+2 k_{2}^{h g^{s}} \beta_{h h}^{g^{s}}\right)\right)
\end{gathered}
$$




\section{References}

Angelitos G, Pavan A (2007) Efficient use of information and social value of information. Econometrica 75(4):1103-1142

Bulow J, Klemperer P (2002) Prices and the winners Curse. Rand J Econ 33(1):1-21

Gal-Or E (1985) Information sharing in oligopoly. Econometrica 53:329-343

Galeotti A, Ghiglino C, Squintani F (2009) Strategic information transmission in networks. J Econ Theory 148(5):1751-1769

Hagenbach J, Koessler F (2009) Strategic communication networks. Rev Econ Stud 77:1072-1099

Kirby AJ (1988) Trade associations as information exchange mechanisms. RAND J Econ 19:138-146

Jackson MO, Wolinsky A (1996) A strategic model of social and economic networks. J Econ Theory 71:44-74

Levin D (2004) The competitiveness of joint bidding in multi-unit uniform-price auctions. Rand J Econ 35(2):373-385

Li L (1995) Cournot oligopoly with information sharing. Rand J Econ 16:521-536

Malueg D, Tsutsui SO (1996) Coalition proof information exchanges. J Econ 63:259-278

Morris S, Shin HS (2002) Social value of public information. Am Econ Rev 92(5):1521-1534

Novshek W, Sonnenschein H (1982) Fulfilled expectations Cournot duopoly with information acquisition and release. Bell J Econ 13:214-218

Radner R (1962) Team decision problems. Ann MathStat 33:857-881

Raith M (1996) A general model of information sharing in oligopoly. J Econ Theory 71:260-288

Ray D, Vohra R (1999) Coalitional power and public goods. J Pol Econ 109(6):1355-1384

Tan X (2012) Two-dimensional values and information sharing in auctions. Mimeo, New York

Vives X (1985) Duopoly information equilibrium: Cournot and Bertrand. J Econ Theory 34:71-94

Ziv A (1993) Information sharing in oligopoly: the truth telling problem. Rand J Econ 24:455-465 